\title{
Benchmarking Measures of Network Controllability on Canonical Graph Models
}

\author{
Elena Wu-Yan'1,2 * Richard F. Betzel ${ }^{2}$. \\ Evelyn Tang ${ }^{2}$. Shi Gu$^{2}$. Fabio Pasqualetti ${ }^{3}$. \\ Danielle S. Bassett ${ }^{2,4,5,6}$
}

Received: 21 September 2017 / Accepted: 5 February 2018 / Published online: 9 March 2018 (C) The Author(s) 2018. This article is an open access publication

\begin{abstract}
The control of networked dynamical systems opens the possibility for new discoveries and therapies in systems biology and neuroscience. Recent theoretical advances provide candidate mechanisms by which a system can be driven from one pre-specified state to another, and computational approaches provide tools to test those mechanisms in real-world systems. Despite already having been applied to study network systems in biology and neuroscience, the practical performance of these tools and associated measures on simple networks with pre-specified structure has yet to be assessed. Here, we study the behavior of four control metrics (global, average, modal, and boundary controllability) on eight canonical graphs (including Erdős-Rényi, regular, small-world, random geometric, Barábasi-Albert preferential attachment, and several modular networks) with different edge weighting schemes
\end{abstract}

Communicated by Paul Newton.

Electronic supplementary material The online version of this article (https://doi.org/10.1007/s00332018-9448-z) contains supplementary material, which is available to authorized users.

$凶$ Danielle S. Bassett

dsb@seas.upenn.edu

1 Department of Computer and Information Sciences, University of Pennsylvania, Philadelphia, PA 19104, USA

2 Department of Bioengineering, University of Pennsylvania, Philadelphia, PA 19104, USA

3 Department of Mechanical Engineering, University of California, Riverside, CA, USA

4 Department of Electrical and Systems Engineering, University of Pennsylvania, Philadelphia, PA 19104, USA

5 Department of Neurology, Hospital of the University of Pennsylvania, Philadelphia, PA 19104, USA

6 Department of Physics \& Astronomy, University of Pennsylvania, Philadelphia, PA 19104, USA 
(Gaussian, power-law, and two nonparametric distributions from brain networks, as examples of real-world systems). We observe that differences in global controllability across graph models are more salient when edge weight distributions are heavy-tailed as opposed to normal. In contrast, differences in average, modal, and boundary controllability across graph models (as well as across nodes in the graph) are more salient when edge weight distributions are less heavy-tailed. Across graph models and edge weighting schemes, average and modal controllability are negatively correlated with one another across nodes; yet, across graph instances, the relation between average and modal controllability can be positive, negative, or nonsignificant. Collectively, these findings demonstrate that controllability statistics (and their relations) differ across graphs with different topologies and that these differences can be muted or accentuated by differences in the edge weight distributions. More generally, our numerical studies motivate future analytical efforts to better understand the mathematical underpinnings of the relationship between graph topology and control, as well as efforts to design networks with specific control profiles.

Keywords Network control theory - Brain networks · Average controllability · Modal controllability $\cdot$ Boundary controllability $\cdot$ Network topology

Mathematics Subject Classification $93 \cdot 62 \cdot 37 \cdot 15$

\section{Introduction}

Complex systems can be modeled as networks in which the system's elements and their pairwise interactions are represented, respectively, as nodes and edges in a graph (Newman et al. 2011). Drawing on a subfield of mathematics known as graph theory, network analysis allows for the quantification of a system's topological organization and offers insight into its function. Network models and associated graph representations have been adopted in a range of disciplines to successfully investigate the structure and function of social, economic, and biological systems (Carrington et al. 2005; Schweitzer et al. 2009; Goh et al. 2007).

Complex systems are also dynamic, meaning that their elements can be associated with internal states that evolve and fluctuate over time (Boccaletti et al. 2006; Porter and Gleeson 2014). The state of an element is system dependent and can correspond to any number of real-world observables, including disease status (e.g., "healthy" or "infected") (Newman 2002; Colizza et al. 2006), the concentration of nutrients at a particular site (Heaton et al. 2012; Papadopoulos et al. 2016), the electrical activity of neurons (Bettencourt et al. 2007; Teller et al. 2014; Wiles et al. 2017), or hemodynamic activity of brain areas (Goñi et al. 2014; Honey et al. 2007). The evolution of a system's state over time depends upon the organization of its underlying network. For example, in social systems, individuals become exposed to and infected by disease through their social contacts (Ray et al. 2016). Similarly, in neural systems, activity propagates from neuron-to-neuron or region-to-region along axonal projections and fiber bundles (Muldoon et al. 2016).

An important question and one that has been the topic of many recent inquiries (Liu et al. 2011; Yan et al. 2012; Sun and Motter 2013; Pasqualetti et al. 2014; Ruths 
and Ruths 2014) is whether a networked dynamical system can be controlled (Liu and Barabási 2016). Network control refers to the prospect of selectively influencing the evolution of a system's state by introducing time-varying external input(s) that drive it from one state to another along a particular trajectory. Current efforts are actively tackling diverse challenges in developing a framework to determine whether control is theoretically possible for a given system (Liu et al. 2011), identifying nodes and edges that are important for efficient control (Kim et al. 2018), and proposing realistic strategies for enacting control over complex systems (Cornelius et al. 2013).

One particularly important approach involves quantifying the contributions of control points (nodes) in driving a system through state space (Pasqualetti et al. 2014). Based on the topological organization of a network as measured by the configuration of edges, certain nodes may be predisposed to drive the system in particular manners based on control strategies (see Fig. 1). For example, some nodes might be better at driving the system into a multitude of different, easy-to-reach states (average controllability), while others may be well-suited for driving the system into difficult-toreach states (modal controllability). Still others are situated between different modules and therefore have the capacity to regulate and control inter-modular synchronization (boundary controllability). These measures of controllability can be made on virtually any networked system, but have been applied most successfully to study large-scale human brain networks (Gu et al. 2015; Tang et al. 2017). These early studies demonstrated that brain systems that support different types of function are also characterized by unique controllability profiles and that these profiles follow distinct trajectories across late development. Despite their application to the study of real-world brain networks, the behavior of average, modal, and boundary controllability measures in practical contexts on canonical network models has not been explored. Such an investigation would help contextualize the behavior of these measures on real-world networks.

In this report, we study the behavior of four metrics that characterize overall controllability (global) as well as distinct control strategies (average, modal, and boundary controllability) in common graph models. We choose these models to canvas architectures that are proven benchmarks in the analysis of complex systems generally, and also to probe architectures that are particularly relevant for neural networks in human and non-human species, a question of critical importance in recent studies (Klimm et al. 2014; Samu et al. 2014; Roberts et al. 2016; Henderson and Robinson 2011; Betzel et al. 2017). This rationale motivated our study of graph ensembles drawn from random, regular lattice, small-world, random geometric, and several modular graph models with differing module size. Because many real-world networks are better characterized by weighted graphs than binary graphs, including brain networks (Bassett and Bullmore 2016), we assigned weights to the edges in each of these models by drawing from theoretically defined distributions such as Gaussian and power-law, as well as from empirically-measured distributions including the fractional anisotropy along white matter tracts in the human brain, or the number of streamlines tracked between brain regions. Our goal is to numerically describe the natural variation of controllability across canonical graph models and to compare that variation to characteristics of brain networks observed in previous studies; we note that a direct comparison between any specific empirical brain network and a relevant model network is left for future 


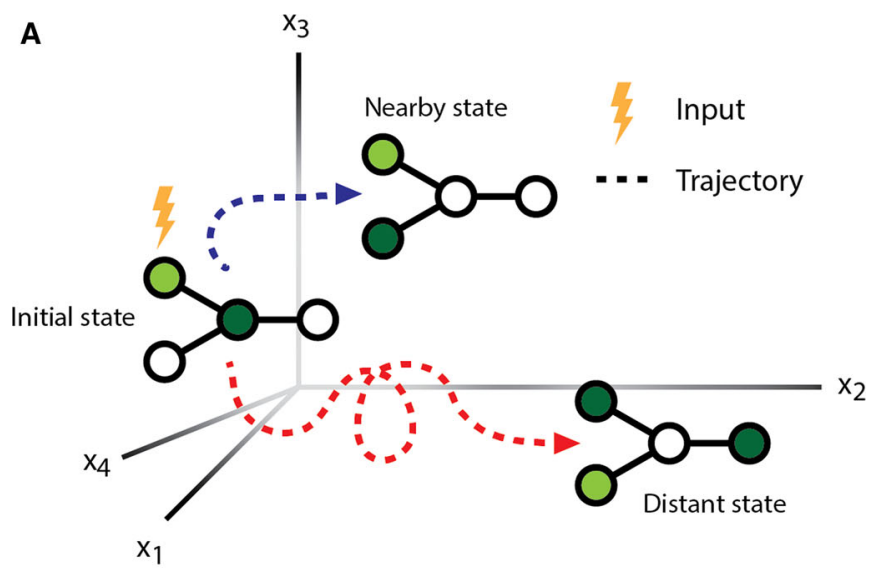

B Average controllers move system into nearby, easy-to-reach states
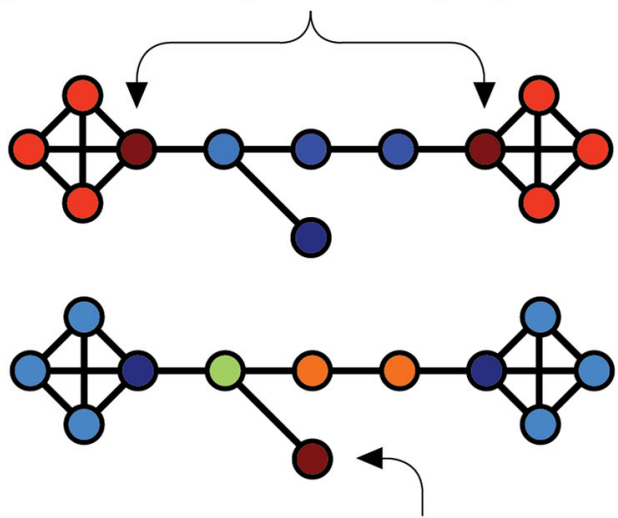

Modal controllers move system into distant, difficult-to-reach states

Boundary controllers help synchronize modules.

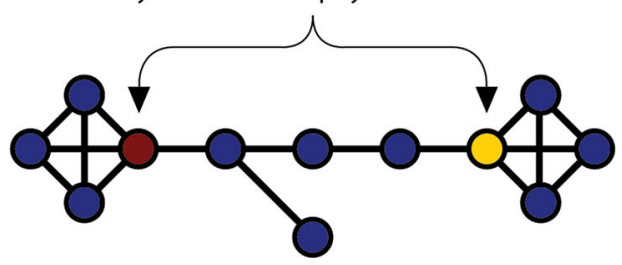

Fig. 1 Network control theory and metrics to describe control strategies. a Controlling a network corresponds to taking a network from a given state (defined as a pattern of node activation magnitudes) to another state (a different pattern of node activation) by applying a control input to a particular node (or set of nodes) in the network. The set of states that is traversed from initial state to target state is known as the trajectory. The target state can be either nearby or distant in energy, and control input is placed at different nodes to effect these two different control strategies. b Metrics to quantitatively characterize different control strategies include average, modal, and boundary controllability. (Top) Average controllability identifies nodes that can move the system into nearby easy-to-reach states with little control energy. (Middle) Modal controllability identifies nodes that can move the system into distant, difficult-to-reach states. (Bottom) Boundary controllability identifies nodes that can help to synchronize or desynchronize communities in the network 
work. More generally, and beyond the narrow domain of brain networks, these choices provide a multi-dimensional space in which to gain generic understanding for how control strategy, graph topology, and edge weight distribution mutually depend on one another, thereby informing future efforts to design networks with specific control profiles.

\section{Materials and Methods}

\subsection{Network Generation}

\subsubsection{Network Definition}

Following common parlance, we will use the terms graph and network interchangeably to refer to a set of nodes connected by edges. Mathematically, we represent a graph $G$ as $G=(V, E)$, where $V$ is the set of vertices, or nodes, and $E$ is the set of edges between nodes. Computationally, we represent graphs as adjacency matrices. A graph or network with $|V|=N$, or $N$ nodes, has an adjacency matrix $A$ of size $N \times N$, where each element $A_{i j}$ denotes the connection strength, or edge weight, between node $i$ and node $j$. We study undirected graphs represented by symmetric adjacency matrices, where $A_{i j}=A_{j i}$, with no self-loops-connections of a given node with itself-i.e., for all $i, A_{i i}=0$. Finally, we distinguish between (i) a binary graph, which only has edge weight values of 0 or 1 to denote the absence or presence, respectively, of a connection between nodes, and (ii) a weighted graph, which can have scaled edge weight values denoting the strength of node-node relations.

\subsubsection{Network Models}

Historically, the fields of graph theory and network science have found it useful to define specific models of graph architectures based on rules for wiring, rules for growth, rules for pruning, and rules for explaining patterns of missing data. In each case, such models provide the grounds for developing and benchmarking novel network statistics. Here, we take a few of the most common graph models from the general literature, as well as more specifically from the literature postulating models of the topology observed in human brain networks (Klimm et al. 2014; Samu et al. 2014; Roberts et al. 2016; Henderson and Robinson 2011), and use them to benchmark the behaviors of recently defined measures of network controllability (Pasqualetti et al. 2014). Below, we briefly describe the eight graph models that we chose as the basis for our analysis of controllability.

- (WRG) Weighted Random Graph model: Arguably the most fundamental, this graph model is a weighted version of the canonical Erdős-Rényi model. For all pairs of $N$ nodes, we modeled the weight of the edge by a geometric distribution with probability of success $p$, the desired edge density of the graph. Each edge weight was assigned the number of successes before the first failure (Sizemore et al. 2017). 
- (RL) Ring Lattice model: In contrast to the random nature of the WRG, the ring lattice model is one with strict order. We arranged $N$ nodes on the perimeter of a regular polygon, each with degree $k$, determined by the desired edge density. We then connected each node to the $\frac{k}{2}$ nodes directly before and after it in the sequence of nodes on the polygon. Edge weights were assigned the inverse of the path length between the two nodes, determined by traversing the perimeter of the polygon (Sizemore et al. 2017).

- (WS) Watts-Strogatz model: A model that bridges both the order of the RL and the disorder of the WRG, the Watts-Strogatz graph model is a ring lattice model in which edges are rewired uniformly at random to create a small-world network. Following (Sizemore et al. 2017), we chose the probability $r$ of rewiring a given edge to maximize the small-world propensity (Muldoon et al. 2016).

- (MD2) Modular Network with 2 communities model: While the previous models can display some local clustering structure, they lack mesoscale organization in the form of modules or communities. In contrast, the Modular Network with 2 communities model is a graph of $N$ nodes and $K$ edges placed so as to form 2 communities. Pairs of nodes within communities are connected with edge density 0.8 , and pairs of nodes between communities (where one node in the pair is in one community and the other node in the pair is in a different community) are connected to fulfill the desired total edge density $p$. We assigned weights to existing edges by considering a geometric distribution with probability of success $p$ if the nodes were in the same module and $1-p$ if the nodes were in different modules. Each edge weight was assigned the number of successes before the first failure (Sizemore et al. 2017).

- (MD4) Modular Network with 4 communities model: This model is generated in a manner identical to that used in the MD2 graph model, with the exception that MD4 has 4 communities.

- (MD8) Modular Network with 8 communities model: This model is generated in a manner identical to that used in the MD2 graph model, with the exception that MD8 has 8 communities.

- (RG) Random Geometric model: In contrast to most of the previous graph models that were agnostic to any embedding space, the Random Geometric model contains $N$ nodes, chosen randomly from a unit cube, and edges whose weights were equal to the inverse of the Euclidian distance between two nodes. We kept only the $K$ shortest edges, in order to maintain the desired edge density $p$ (Sizemore et al. 2017).

- (BA) Barábasi-Albert model: In our final graph model, we use software from Klimm et al. (2014) to generate a typical BA model-a scale-free network that exhibits preferential attachment to existing nodes of high degree-with $N$ nodes and $K$ edges. Each edge weight was assigned the average degree of the two nodes it connected.

While of course this is not an exhaustive list of the possible graph models that one might wish to study, we focus on this set because it provides a useful assessment of quite different topologies, and because most of these models have been suggested as relevant benchmarks against which to compare brain networks in previous studies. 


\subsubsection{Network Size}

In all of the graph models described above, two parameters must be fixed a priori: the number of nodes $N$ in the network, and the number of edges $K$ in the network. We chose the number of nodes to be 128 (see Results section), and we confirmed consistency of our findings across two other network sizes (256, or 512, nodes; see Supplementary Materials). We chose the number of edges to produce network densities that were consistent with those observed empirically in large-scale human brain graphs. Specifically, drawing on recently published data from 30 healthy adult individuals by capitalizing on a 19-minute multi-band diffusion spectrum imaging sequence (Betzel et al. 2016), we assigned the 128-node graphs an edge density of 0.2919, the 256-node graphs an edge density of 0.2175 , and the 512-node graphs an edge density of 0.1396 . For each network size, we generated 100 instantiations of each of the 8 graph models described above.

\subsubsection{Network Weighting}

All of the eight graph models described above were weighted graph models (Sizemore et al. 2017). While it is important to study weighted (as opposed to binary) graph models to benchmark network controllability statistics that are currently being applied to real-world weighted graphs, comparisons across models are confounded by the fact that each model can have a very different edge weight distribution. Here, we sought to disentangle the impact of graph model from the impact of edge weight distribution on network controllability statistics. Practically, we therefore developed a pipeline to reweight all of the graph models fairly, and with a fixed edge weight distribution.

We began by adding random noise on the order of $10^{-7}$ to all edge weights in all network models; this process ensures the uniqueness of each edge weight, while maintaining the relative weight magnitudes. Next, we sorted edges by weight and then replaced each edge with corresponding ordered values pulled from a specific edge weight distribution of interest, of which we defined four. The first was a Gaussian distribution with a mean of 0.5 and a standard deviation of 0.12 . The second was a power-law distribution with a slope of -3 and a range of values from $10^{-5}$ to $10^{5}$. Both Gaussian and power-law distributions are ubiquitously found in real-world networks and in fact form natural benchmarks for edge weight distributions taken from neuroimaging data. The third and fourth edge weight distributions of interest were taken from Betzel et al. (2016) to closely model empirical weighting distributions in large-scale human brain structural networks estimated from diffusion imaging tractography. Specifically, these two distributions were streamline counts (normalized by the geometric mean of regional volumes) and fractional anisotropy (FA). Importantly, the reweighting scheme we describe here allowed us to use the same edge weighting across all graphs to guarantee that differences in controllability were due to topology and not to other properties of the graphs, like differing edge weights and scaling.

\subsubsection{Network Ensembles}

In summary, we study three network sizes $(128,256$, or 512 nodes) for each of the four edge weightings (Streamline Counts, FA, Gaussian, Power-Law), thus totaling 
12 sets of networks, each of which included 100 instantiations of each of the 8 graph models. We next turn to an examination of network controllability statistics in these 12 sets of weighted graphs.

\subsection{Network Controllability}

\subsubsection{A Linear Model of Brain Dynamics}

Although the relationship of brain structural networks to the correlation structure of spontaneous neural activity is, in general, nonlinear, a great deal of variance in that correlation structure can, nonetheless, be explained by simple linear models (Galán 2008). Accordingly, we define brain dynamics with a noise-free, linear, discrete-time, and time-invariant network model:

$$
\mathbf{x}(t+1)=\mathbf{A x}(t)+\mathbf{B}_{\mathcal{K}} \mathbf{u}_{\mathcal{K}}(t)
$$

where $\mathbf{x}: \mathbb{R}_{\geq 0} \rightarrow \mathbb{R}^{N}$ denotes a brain region's state (i.e., its magnitude of electrical or hemodynamic activity) and $\mathbf{A}$ is the symmetric, weighted adjacency matrix. The input matrix, $\mathbf{B}_{\mathcal{K}}$, which identifies control points $\mathcal{K}=\left\{k_{1}, \ldots, k_{m}\right\}$, is defined as:

$$
\mathbf{B}_{\mathcal{K}}=\left[e_{k_{1}}, \ldots, e_{k_{m}}\right]
$$

where $e_{i}$ indicates the $i$-th canonical vector (of length $N$ ). The input, $\mathbf{u}_{\mathcal{K}}: \mathbb{R}_{\geq 0} \rightarrow \mathbb{R}^{m}$, specifies the strategy for controlling these dynamics. Note that in Eq. 1, we scaled the elements of $\mathbf{A}$ by $1 /\left(1+\lambda_{\max }\right)$ (where $\lambda_{\max }$ is the largest eigenvalue of unscaled $\mathbf{A}$ ), which ensures that the scaled version of $\mathbf{A}$ is Schur stable (i.e., all eigenvalues of $\mathbf{A}$ are $<1$ in magnitude). We note that different choices for the normalization will accentuate versus de-emphasize different scales of dynamics, and it will be interesting in the future to study how the choice of normalization impacts observed patterns of controllability.

Under these dynamics, we define a series of control metrics that quantify different intuitions of controllability (although see "Methodological Considerations" for a discussion of other interesting metrics not studied here). Specifically, we will study global, average, modal, and boundary controllability. Global and average controllability are defined for arbitrary $\mathbf{A}$ and $\mathbf{B}$, while modal and boundary controllability are defined for each node in the network.

\subsubsection{Global Controllability}

The first metric we will study is global controllability, which assesses whether control (i.e., the ability to steer the system from any arbitrary network state to any other arbitrary network state) is theoretically possible. In general, the ease or difficulty of control is related to the structure and eigenvalues of the controllability Gramian: 


$$
\mathbf{W}_{\mathcal{K}}=\sum_{t=0}^{\infty} \mathbf{A}^{t} \mathbf{B}_{\mathcal{K}} \mathbf{B}_{\mathcal{K}}^{T} \mathbf{A}^{t}
$$

where the eigenvalues of the inverse Gramian indicate ease of control. We define global controllability to be the smallest eigenvalue of $\mathbf{W}_{\mathcal{K}}$. The structure of $\mathbf{W}_{\mathcal{K}}$ depends upon both the topological organization of a network (which we assume to be fixed) and the set of nodes assumed to be control points, i.e., $\mathbf{B}_{\mathcal{K}}$. As in $\mathrm{Gu}$ et al. (2015), we test all possible single-point control strategies (where $\mathbf{B}_{\mathcal{K}}$ is a one-dimensional column vector), computing $\mathbf{W}_{\mathcal{K}}$ and its corresponding eigenvalues along with the global controllability metric for each case. Accordingly, for every realization of any graph model, we obtain $N$ global controllability scores for each of the $N$ nodes. We note that in practice, we find that-for the networks that we study here- - the largest eigenvalue of the Gramian is greater than or equal to 1 . Thus, the global controllability values that we report later in the Results section are small in comparison to the largest eigenvalue.

\subsubsection{Average and Modal Controllability}

In addition to global controllability, we also characterized networks using three other node-level controllability metrics. The first, average controllability, $\zeta$, describes the ease with which control points in a network can move the system into nearby, easily reachable states, and is computed as

$$
\zeta=\operatorname{Trace}\left(\mathbf{W}_{\mathcal{K}}^{-1}\right)
$$

The second metric, modal controllability, quantifies the ease with which control points in a network can move the system into distant, hard-to-reach states. Modal controllability is computed from the eigenvector matrix $V=\left[v_{i j}\right]$ of the network adjacency matrix A (and not from the controllability Gramian). By extension from the PBH test (Kailath 1980), if the entry $v_{i j}$ is small, then the $j$-th mode is poorly controllable from node $i$. Following (Pasqualetti et al. 2014), we define

$$
\phi_{i}=\sum_{j}\left(1-\xi_{j}^{2}(\mathbf{A})\right) v_{i j}^{2}
$$

as a scaled measure of the controllability of all $N$ modes $\xi_{0}(\mathbf{A}), \ldots, \xi_{N-1}(\mathbf{A})$ from the brain region $i$-allowing the computation of regional modal controllability. Regions with high modal controllability are able to control all the dynamic modes of the network and hence to drive the dynamics toward hard-to-reach configurations.

Past studies have shown that, for a given graph, average and modal controllability tend to be anti-correlated with one another across nodes ( $\mathrm{Gu}$ et al. 2015; Tang et al. 2017), so that brain regions well-suited for moving the system into easy-to-reach states are also poorly suited for moving the system into difficult-to-reach states and vice versa. 


\subsubsection{Boundary Controllability}

Lastly, we also investigated boundary controllability, which describes the ease with which control points in a network can act to decouple or connect communities (subnetworks) within the system. To detect boundary controllers, we followed the procedure described in $\mathrm{Gu}$ et al. (2015), which modified the algorithm proposed in Pasqualetti et al. (2014). Briefly, this procedure involves iteratively bi-partitioning the original network into progressively smaller subnetworks and, at each level, identifying nodes whose connections span both halves. In Pasqualetti et al. (2014), the initial partition of the network into two subnetworks was generated based on the Fiedler eigenvector of $\mathbf{A}$. Here, because brain networks are composed of many distinct communities (Sporns and Betzel 2016), we initialize the algorithm instead with a partition obtained by maximizing a modularity quality function (Newman and Girvan 2004):

$$
Q=\sum_{i j}\left[A_{i j}-P_{i j}\right] \delta\left(\sigma_{i} \sigma_{j}\right),
$$

where $P_{i j}$ is the expected weight of the connection between nodes $i$ and $j, \sigma_{i}$ is the subnetwork (community) assignment of node $i$, and $\delta\left(\sigma_{i}, \sigma_{j}\right)$ is the Kronecker delta function, which equals one when $\sigma_{i}=\sigma_{j}$ and zero otherwise. Here, the expected weight of connections is determined based on a null model in which nodes' strengths are preserved but connections are, otherwise, formed at random: $P_{i j}=\frac{k_{i} k_{j}}{2 m}$, where $k_{i}=\sum_{j} A_{i j}$ and $2 m=\sum_{i} k_{i}$. This null model is commonly known as the NewmanGirvan null model.

From this initial partition, we define boundary nodes to be nodes that maintained supra-threshold connections to more than one subnetwork. The value of this threshold, $\rho=1 \times 10^{-5}$, was selected so as to-in general across graph models and instantiations - maximize the number of unique values of boundary controllability across nodes in a given graph. At this first level, we assign the set of $N_{1}$ boundary nodes the boundary controllability value of 1 . Then, we bi-partitioned subnetworks according to the Fiedler eigenvector, and boundary points were identified as nodes that maintained supra-threshold connections to both subnetworks. We assign to these boundary nodes the boundary controllability value of $\left(N-N_{1}\right) / N$. Finally, we iterate this process until all nodes have been assigned a boundary controllability value. To demonstrate the reliability of our results given reasonable variations in the choice of threshold, we also provide results for $\rho=1 \times 10^{-3}$ and $\rho=1 \times 10^{-8}$ in the Supplementary Materials.

\section{Results}

\subsection{Variation in Network Controllability Statistics Across Graph Models}

We begin by examining how network controllability statistics vary over graphs within a given ensemble and whether that variation differs as a function of graph model. Following the procedure outlined in the Methods section, for each con- 

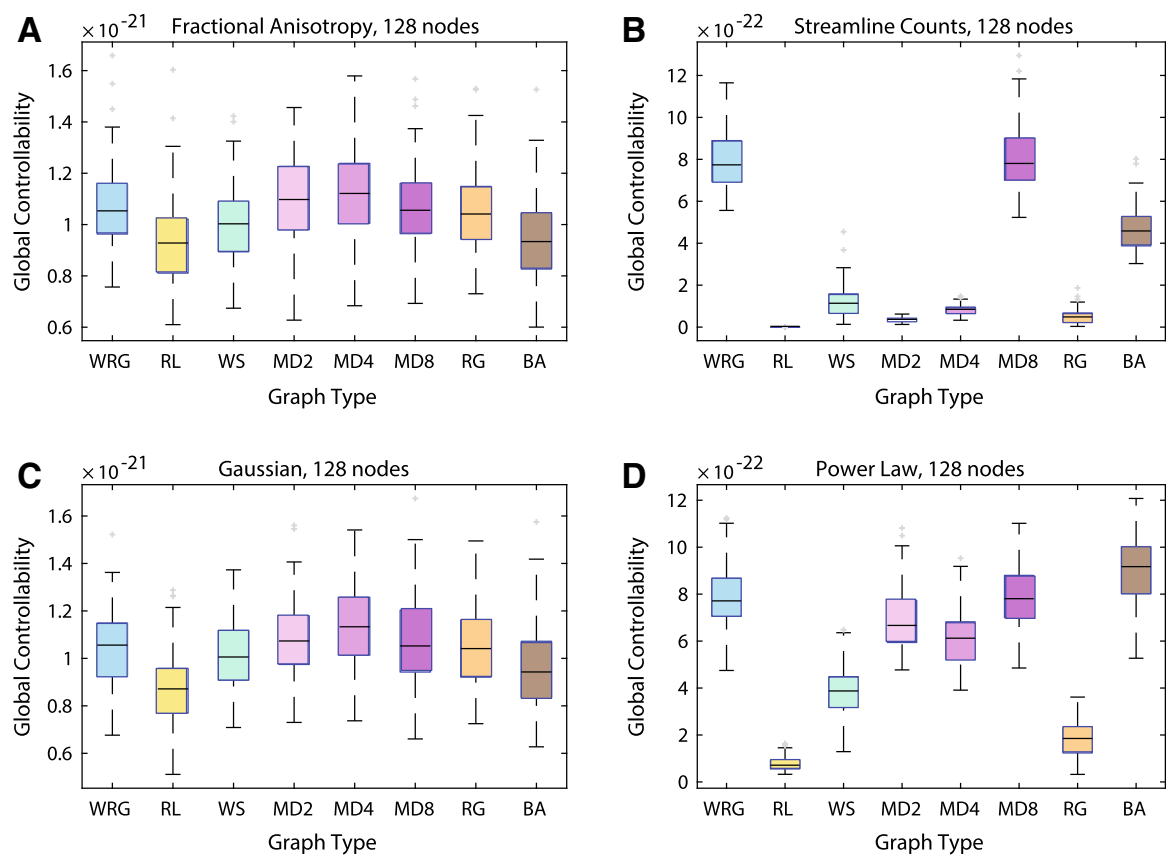

Fig. 2 Variation in global controllability as a function of edge weighting and graph model. Global controllability values were averaged over nodes in each graph model ensemble, and therefore, boxplots show variation over graphs in the ensemble. Results are shown for four edge weighting schemes: a FA, b streamline counts, c Gaussian, d power-law. The eight graph models include the weighted random graph (WRG), the ring lattice (RL), the Watts-Strogatz small-world (WS), the modular graphs (MD2, MD4, MD8), the random geometric $(\mathrm{RG})$, and the Barábasi-Albert preferential attachment (BA) models

trollability type, we took the mean of the 128 sorted controllability values across the $n$ nodes in each graph instance, giving us 100 controllability values averaged over the 128 regions. For each controllability type, we used four identical edge weight distributions corresponding to fractional anisotropy (FA) and streamline counts (SC) from real brain data, a Gaussian distribution, and a power-law distribution.

When considering global controllability for graph models constructed with Gaussian and FA weighting schemes, we observed that all graph types had global controllability values similar in range and high variance, with RL at the low end of the range and MD4 at the high end of the range (Fig. 2a,c). For graph models constructed with streamline counts and power-law weighting schemes, we observed varying behaviors. The SC-weighted RL, WS, MD2, MD4, and RG graphs all had low mean and low variance, while WRG, MD8, and BA had higher mean values and higher variance. The power-law-weighted RL, WS, and RG graphs had lowest means and variance, while the other graph types had higher mean and variance, with the BA having the highest mean and variance (Fig. 2b,d). One-way ANOVAs indicated significant effects of graph type for all of the four edge weight distributions (see Table 1). 

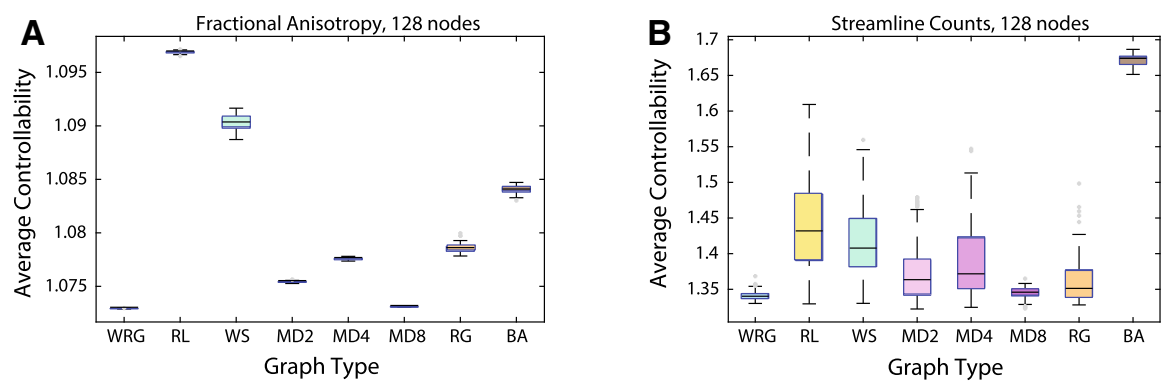

C

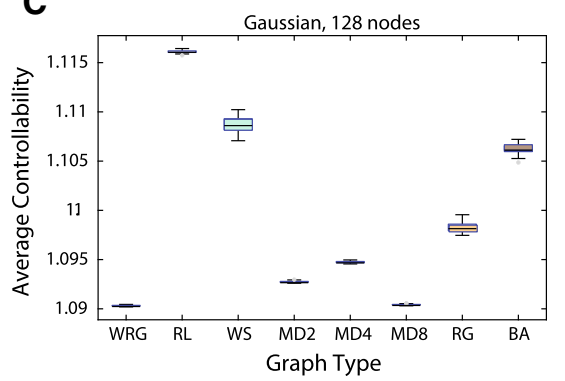

D

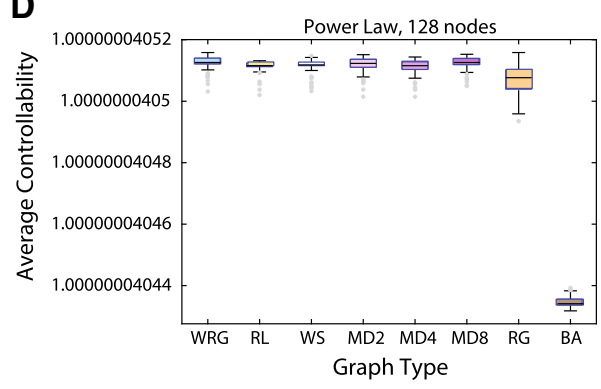

Fig. 3 Variation in average controllability as a function of edge weighting and graph model. Average controllability values were averaged over nodes in each graph model ensemble, and therefore, boxplots show variation over graphs in the ensemble. Results are shown for four edge weighting schemes: a FA, b streamline counts, c Gaussian, $\mathbf{d}$ power-law. The eight graph models include the weighted random graph (WRG), the ring lattice (RL), the Watts-Strogatz small-world (WS), the modular graphs (MD2, MD4, $\mathrm{MD} 8$ ), the random geometric (RG), and the Barábasi-Albert preferential attachment (BA) models

When considering average controllability for graph models constructed with Gaussian and FA weighting schemes, we observed that WRG, MD2, MD4, and MD8 all had similarly low mean average controllability values (between 1.090 and 1.095) with small variance (Fig. 3a,c). RG, BA, WS, and RL then followed in increasing order of mean, and WS had the highest variance. For graph models constructed with the power-law-weighting scheme, we observed relatively uniform behavior, all with high controllability values, low variance, and skewed left-tailed distributions toward lower controllability values (Fig. 3d). The exception was the BA graph, which had low average controllability and low variance. The SC-weighted graphs varied in mean value and variance, with WRG and MD8 having the lowest mean and lowest variance and BA having the highest mean and low variance (Fig. 3c). RG, MD2, MD4, WS, and RL then followed with increasing mean and variance, all with slightly right-tailed distributions. One-way ANOVAs indicated significant effects of graph type for all of the four edge weight distributions (see Table 1).

When considering modal controllability for graph models constructed with Gaussian and FA weighting schemes, we observed that WRG, RL, WS, MD2, MD4, and MD8 all had small mean modal controllability values and low variance (Fig. 4a,c). RG had higher mean controllability values with larger variance and outliers, while BA 

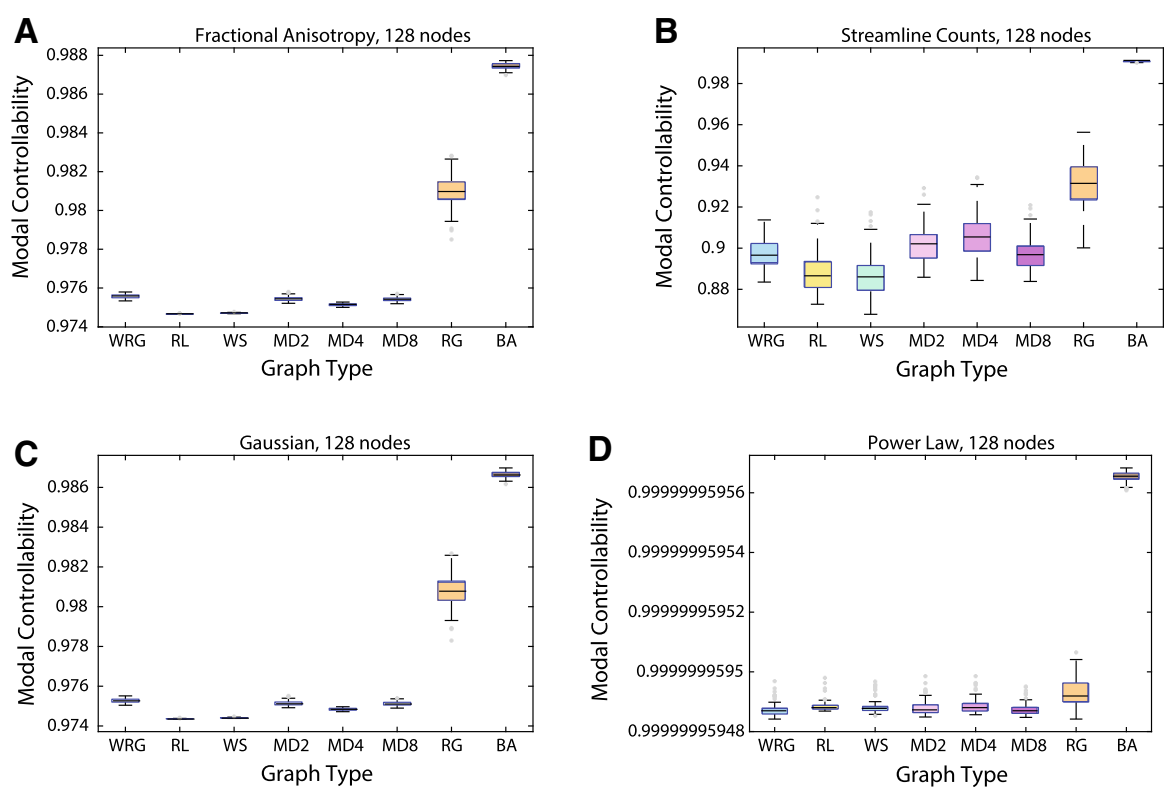

Fig. 4 Variation in modal controllability as a function of edge weighting and graph model. Modal controllability values were averaged over nodes in each graph model ensemble, and therefore, boxplots show variation over graphs in the ensemble. Results are shown for four edge weighting schemes: a FA, b streamline counts, c Gaussian, d power-law. The eight graph models include the weighted random graph (WRG), the ring lattice (RL), the Watts-Strogatz small-world (WS), the modular graphs (MD2, MD4, MD8), the random geometric $(\mathrm{RG})$, and the Barábasi-Albert preferential attachment (BA) models

had the highest mean values with small variance. For graph models constructed with the power-law-weighting scheme, we observed relatively uniform behavior, all with low controllability values, low variance, and skewed right-tailed distributions toward higher controllability values (Fig. 4d). The exception was the BA graph, which had high average controllability and low variance. The SC-weighted graphs varied in mean value (with RL and WS having the lowest mean and BA having the highest mean) but had similar variance with slightly right-tailed distributions (Fig. 4b). One-way ANOVAs indicated significant effects of graph type for all of the four edge weight distributions (see Table 1).

When considering boundary controllability for graph models constructed with the Gaussian weighting scheme, we observed that RL, WS, MD2, and MD4 all had similarly small boundary controllability values and low variance (Fig. 5a). Then, RG and BA had increasingly higher controllability values and variance. MD8 had higher controllability values with small variance and a slightly left-tailed distribution of outliers. WRG had the highest controllability values and relatively small variance. For graph models constructed with the FA weighting scheme, trends were similar but the variance in the mean values for each type of graph tended to be higher and also more right-skewed compared to those of the Gaussian-weighted graphs (Fig. 5c). For graph models constructed with the SC-weighted graphs, we observed relatively uniform behavior, with low mean values (ranging from 0 to 1 ), low variance, and 
Table 1 Effect of graph model on controllability statistics

$\begin{array}{ll}\text { Global } & F(7)=16.41, p=2.8 \times 10^{-20} \\ \text { Fractional anisotropy } & F(7)=23.92, p=1.3 \times 10^{-29} \\ \text { Gaussian } & F(7)=1559.39, p=0.00 \\ \text { Streamline counts } & F(7)=671.62, p=0.00 \\ \text { Power-law } & \\ \text { Average } & F(7)=79446.27, p=0.00 \\ \text { Fractional anisotropy } & F(7)=71074.27, p=0.00 \\ \text { Gaussian } & F(7)=760.92, p=0.00 \\ \text { Streamline counts } & F(7)=11748.73, p=0.00 \\ \text { Power-law } & \\ \text { Modal } & F(7)=22830.9, p=0.00 \\ \text { Fractional anisotropy } & F(7)=21809.02, p=0.00 \\ \text { Gaussian } & F(7)=1525.89, p=0.00 \\ \text { Streamline counts } & F(7)=11765.4, p=0.00 \\ \text { Power-law } & \\ \text { Boundary } & F(7)=10015.69, p=0.00 \\ \text { Fractional anisotropy } & F(7)=10968.61, p=0.00 \\ \text { Gaussian } & F(7)=873.59, p=0.00 \\ \text { Streamline counts } & F(7)=5838.1, p=0.00\end{array}$

Results of one-way ANOVAs assessing the effect of graph model on each controllability statistic, and each edge weighting scheme, as shown in the boxplots in Figs. 2, 3, 4, and 5

right-tailed distributions, with the BA graph having the longest tail (Fig. 5b). The power-law-weighted graphs had similar behavior to the SC-weighted graphs but had higher variance overall (Fig. 5d). One-way ANOVAs indicated significant effects of graph type for all of the four edge weight distributions (see Table 1).

To summarize, when considering trends within a single edge weighting scheme, it is important to note that because edge weight distributions were exactly the same across each of the graph types, differences in controllability are due to network topology rather than the effects of differing edge weights. Since each of the graph types exhibits distinct behavior of controllability values for all types of controllability, this suggests that the topology of a network largely influences global, average, modal, and boundary controllability. Further, when considering trends across edge weighting schemes for a single graph model, it is important to note that this guarantees that differences in controllability are due to the effects of differing edge weights rather than network topology. The similarity of trends in the controllability values between the FA and Gaussian weighting and then between the SC and power-law weighting suggests that the nature of edge weights influences controllability when edge weight distributions are more normal (FA and Gaussian) versus when they are very skewed (SC and powerlaw). 

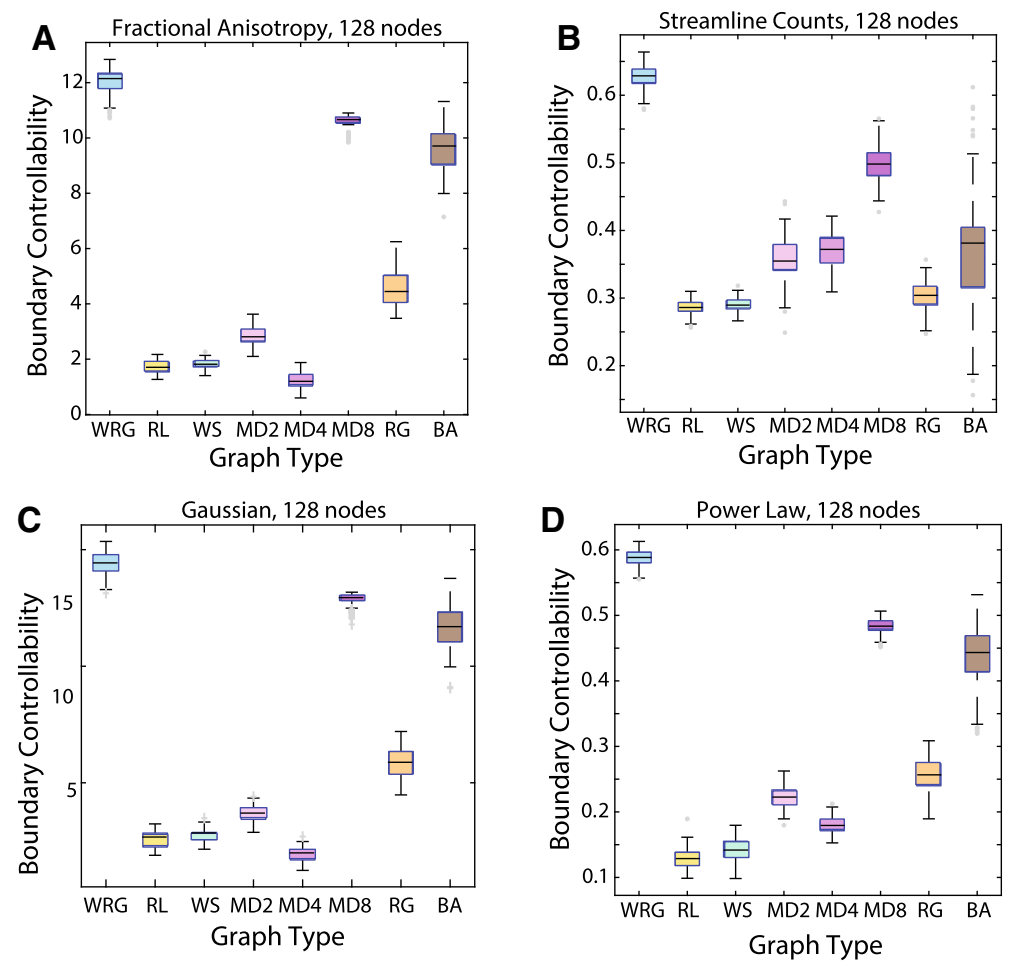

Fig. 5 Variation in boundary controllability as a function of edge weighting and graph model. Boundary controllability values were averaged over nodes in each graph model ensemble, and therefore, boxplots show variation over graphs in the ensemble. Results are shown for four edge weighting schemes: a FA, b streamline counts, $\mathbf{c}$ Gaussian, $\mathbf{d}$ power-law. The eight graph models include the weighted random graph (WRG), the ring lattice (RL), the Watts-Strogatz small-world (WS), the modular graphs (MD2, MD4, MD8), the random geometric (RG), and the Barábasi-Albert preferential attachment (BA) models. Note that these results are presented for $\rho=10^{-8}$; we observe consistent results across thresholds of $10^{-5}, 10^{-8}$, and $10^{-11}$ of edge weight distribution (see Supplement)

\subsection{Relation Between Controllability Statistics Across Graphs}

In prior work, we have observed that average and modal controllability, averaged over nodes, are positively related to one another across brain networks (Tang et al. 2017). These data suggest that brain networks that are structurally predisposed to be effective in moving network dynamics into easy-to-reach states (via average control) are also the brain networks that are structurally predisposed to be effective in moving network dynamics into difficult-to-reach states (via modal control). Here, we ask whether this positive relationship between average and modal controllability across networks holds in canonical graph models.

In the Gaussian weighting scheme, we observed that one of the graphs (MD4) exhibited no correlation between average and modal controllability, while MD8 and RG had a moderate positive correlation, and WRG and BA had nearly linear positive correlations (Fig. 6). Indeed, across other weighting schemes, there was no consistent 

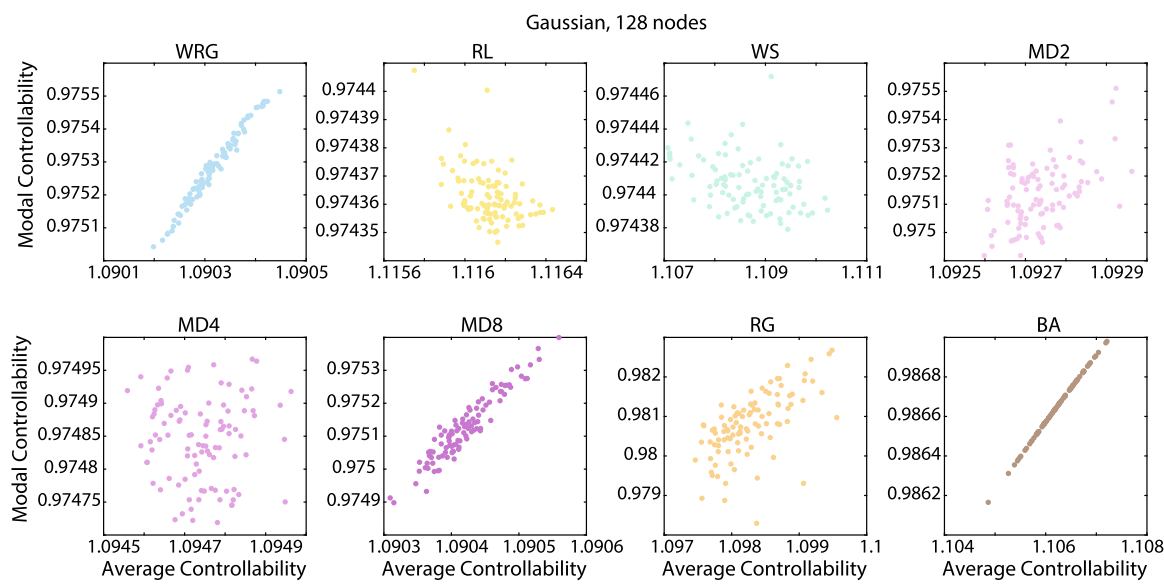

Fig. 6 Relation between average and modal controllability across graphs for the Gaussian edge weighting scheme. Average and modal controllability values were averaged over nodes in each graph model ensemble, and therefore, scatterplots show values for each graph in the ensemble. Results are shown for the Gaussian edge weighting scheme. The eight graph models include the weighted random graph (WRG), the ring lattice (RL), the Watts-Strogatz small-world (WS), the modular graphs (MD2, MD4, MD8), the random geometric $(\mathrm{RG})$, and the Barábasi-Albert preferential attachment (BA) models

positive or negative relation between average and modal controllability (see Supplementary Materials). Specifically, in the FA weighting scheme, we observed that most graphs did not exhibit a strong correlation between average and modal controllability, except for WRG and BA, which had a moderate positive and strong positive correlation, respectively. Interestingly, in the streamline count weighting scheme, we observed that RL, WS, MD2, MD4, and MD8 exhibited a moderate negative correlation, while WRG and RG had weak negative correlations. BA exhibited a nearly linear, strong positive correlation. In the power-law-weighting scheme, all eight graph models exhibited a strong negative correlation between average and modal controllability that was nearly linear. For Spearman $\rho$ correlation coefficients, see Table 2; we note that Gaussian and FA-weighted graphs were less likely to display a significant correlation between average and modal controllability than streamline count and power-law-weighted graphs.

While not previously reported in prior work, we also asked whether boundary controllability statistics were correlated with either average or modal controllability statistics across graphs in an ensemble, after averaging nodal values across all nodes in each graph. In general for Gaussian weighting schemes, we observe that average and modal controllability do not tend to be strongly correlated with boundary controllability across graph instances (Figs. 7 and 8). Across other weighting schemes, we observe the same trends, for no strong relationships between boundary controllability statistics and either average or modal controllability statistics across graphs in an ensemble (Tables 3 and 4). 


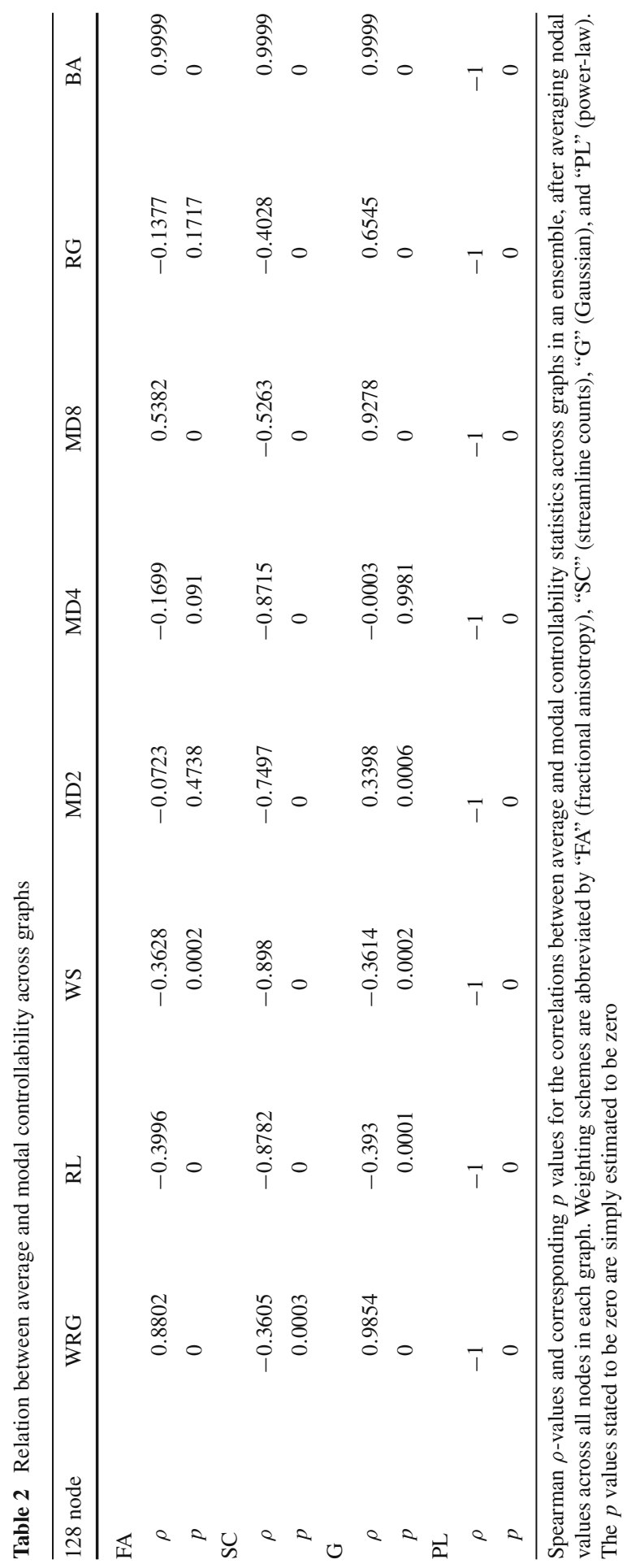



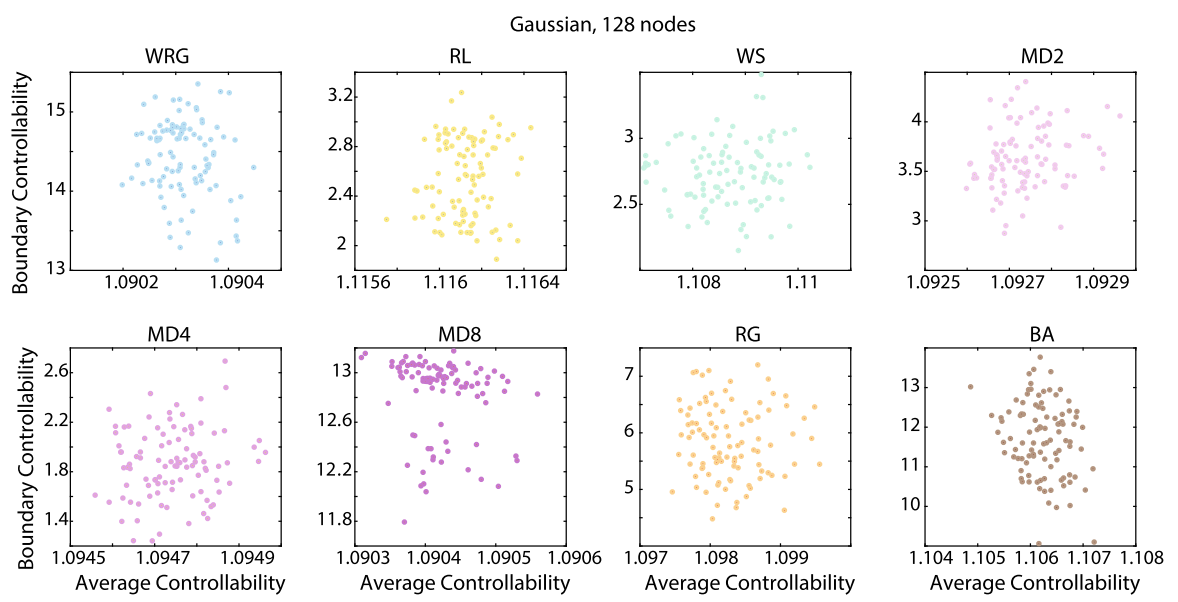

Fig. 7 Relation between average and boundary controllability across graphs for the Gaussian edge weighting scheme. Average and boundary controllability values were averaged over nodes in each graph model ensemble, and therefore, scatterplots show values for each graph in the ensemble. Results are shown for the Gaussian edge weighting scheme. The eight graph models include the weighted random graph (WRG), the ring lattice (RL), the Watts-Strogatz small-world (WS), the modular graphs (MD2, MD4, MD8), the random geometric (RG), and the Barábasi-Albert preferential attachment (BA) models
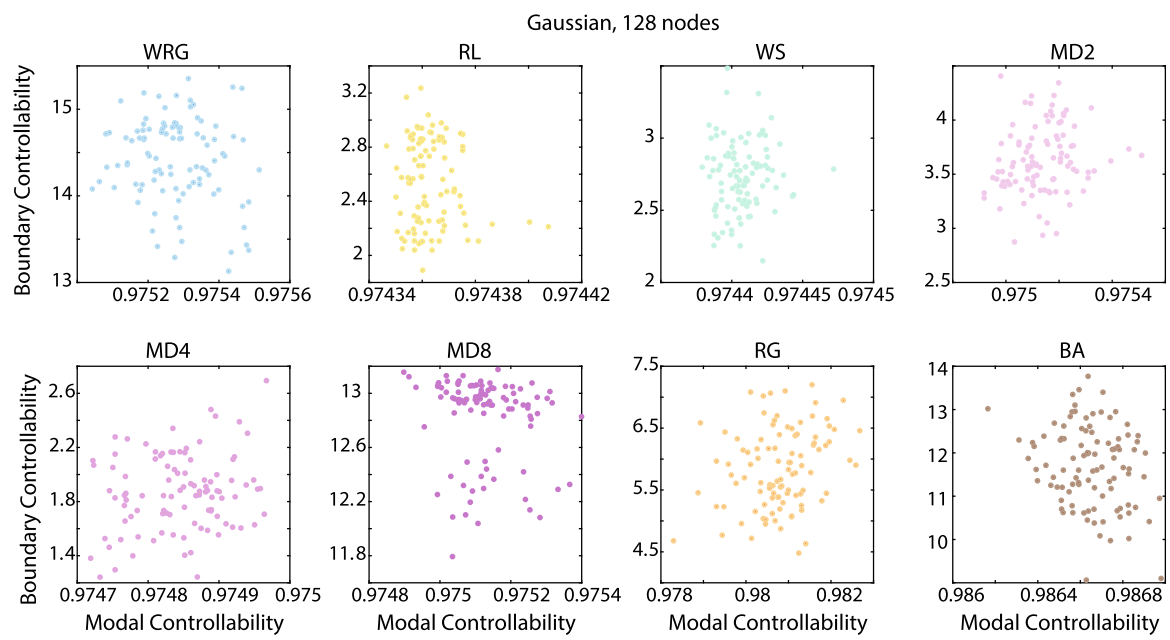

Fig. 8 Relation between modal and boundary controllability across graphs for the Gaussian edge weighting scheme. Modal and boundary controllability values were averaged over nodes in each graph model ensemble, and therefore, scatterplots show values for each graph in the ensemble. Results are shown for the Gaussian edge weighting scheme. The eight graph models include the weighted random graph (WRG), the ring lattice (RL), the Watts-Strogatz small-world (WS), the modular graphs (MD2, MD4, MD8), the random geometric (RG), and the Barábasi-Albert preferential attachment (BA) models 


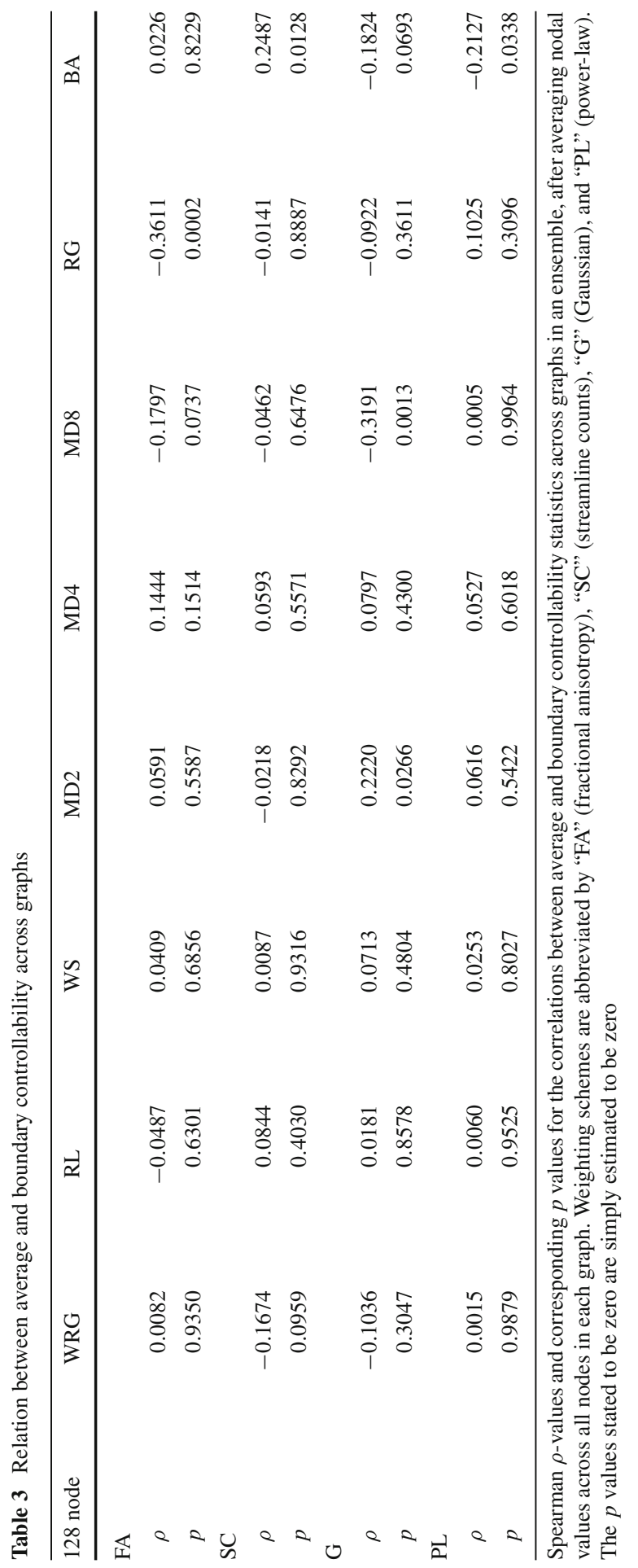




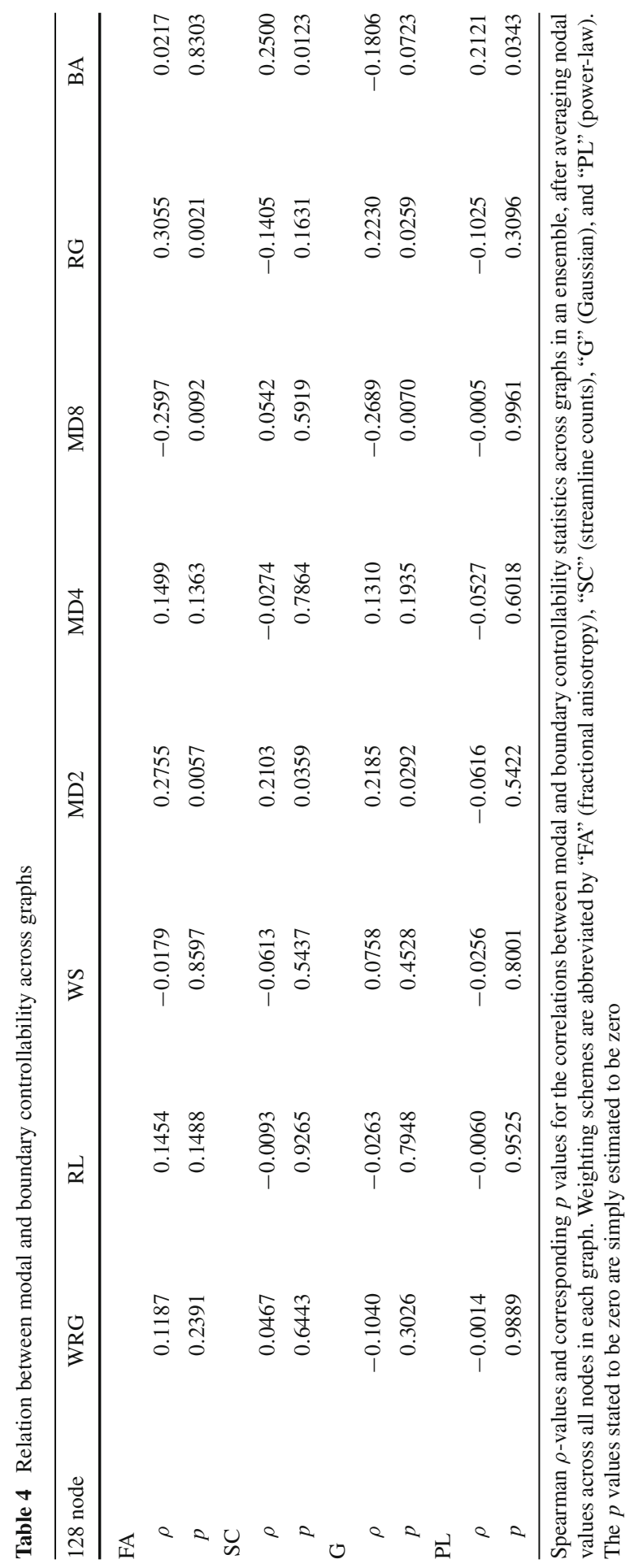




\subsection{Effect of Graph Size on Nodal Variation in Network Controllability Statistics Across Graph Models}

To assess the reliability and reproducibility of our results, we next examined the impact of graph size ( $n=128,256$, or 512$)$ on network controllability statistics, and their modulation by graph model for the Gaussian edge weight distribution (see Supplementary Materials). We observed that global, average, modal, and boundary controllability values for graphs of 256 and 512 nodes largely maintained the same trends as for graphs with 128 nodes. However, mean global, average, and modal controllability values tended to decrease with increasing graph size (consistent with analytical studies), while mean boundary controllability values tended to increase with increasing graph size (with the exception of MD8). Variance of average and modal controllability tended to decrease with increasing graph size. Importantly, because size varied within the same type of controllability for the same set of graphs, this guarantees that differences in controllability are due to the effects of differing network size rather than network topology. The similarity of trends across graph sizes suggests that the controllability properties are maintained in networks of different sizes but may be accentuated through decreased spread or mean value with increasing size.

\subsection{Nodal Variation in Network Controllability Statistics Across Graph Models}

In the previous section, we examined how network controllability statistics varied over graphs within a given ensemble and whether that variation differed as a function of graph model. These questions focused on values of controllability statistics that were calculated at each node of the network separately and then averaged over nodes in the network. In this section, we turn to an examination of the nodal variation in network controllability statistics and ask questions regarding how nodal variation differs across graph models and between controllability statistics. Following the procedure outlined in the Methods section, for each controllability type we took the mean of the 128 sorted nodal controllability values across all 100 graphs in a given model ensemble, giving us 128 controllability values averaged over the graph instances. For each controllability type, we used four identical edge weight distributions corresponding to fractional anisotropy (FA) and streamline counts (SC) from real brain data, Gaussian distribution, and power-law distribution.

When considering global controllability for graph models constructed with Gaussian and FA weighting schemes, we observed that all graph models had nearly identical right-tailed distributions with primarily low global controllability values and low variance (Fig. 9a,c). For graph models constructed with streamline counts and power-law-weighting schemes, we observed a similar pattern but with generally lower variance for graphs such as RL, WS, and RG (as well as MD2 and MD4 for the streamline count weighting) and slightly less skew toward higher values (Fig. 9b,d). One-way ANOVAs indicated significant effects of graph type for all of the four edge weight distributions (see Table 5).

When considering average controllability for graph models constructed with Gaussian and FA weighting schemes, we observed that WRG, MD2, MD4, and MD8 all 

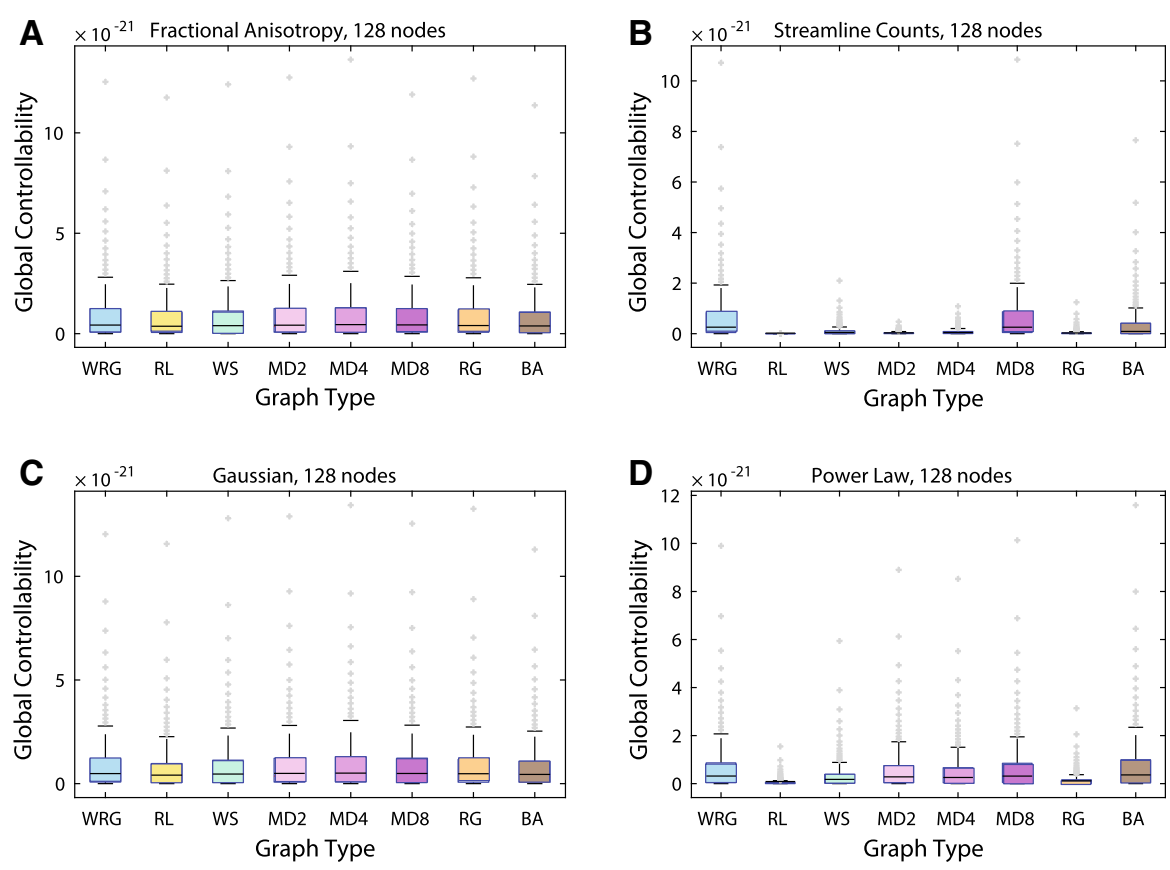

Fig. 9 Nodal variation in global controllability as a function of edge weighting and graph model. Global controllability values were averaged over instances in each graph model ensemble, and therefore, boxplots show variation over nodes in the graph. Results are shown for four edge weighting schemes: a FA, b streamline counts, c Gaussian, d power-law. The eight graph models include the weighted random graph (WRG), the ring lattice (RL), the Watts-Strogatz small-world (WS), the modular graphs (MD2, MD4, MD8), the random geometric (RG), and the Barábasi-Albert preferential attachment (BA) models

had similar mean average controllability values with low variance (Fig. 10a,c). RL and WS had slightly higher mean average controllability values and the lowest variance of all the graph types. RG and BA had the lowest mean average controllability values and highest variance, and the BA model was also most skewed, with a significant right-tailed distribution toward higher controllability values. In contrast, the graphs constructed with power-law and streamline count weighting schemes exhibited relatively uniform behavior, all with low controllability values, low variance, and skewed right-tailed distributions toward higher controllability values (Fig. 10b,d). One-way ANOVAs indicated significant effects of graph type for three of the four edge weight distributions: FA, streamline counts, and Gaussian but not power-law (see Table 5).

When considering the modal controllability for graph models constructed with Gaussian and FA weighting schemes, we observed that WRG, RL, WS, MD2, MD4, and MD8 all had similar mean modal controllability values and similar variance (Fig. 11a,c). RG had a higher mean controllability value, while BA had the highest. RL and WS had the smallest variance, while RG and BA had the largest variance. The BA model was also the most skewed, with a significant left-tailed distribution toward lower controllability values. In contrast, the graphs constructed with power-law and streamline count weighting schemes exhibited nearly uniform behavior, all with high 
Table 5 Effect of graph model on nodal controllability statistics

\begin{tabular}{ll}
\hline Global & $F(7)=16.08, p=3.6 \times 10^{-20}$ \\
Fractional anisotropy & $F(7)=23.31, p=1.8 \times 10^{-29}$ \\
Gaussian & $F(7)=851.46, p=0.00$ \\
Streamline counts & $F(7)=651.08, p=0.00$ \\
Power-law & \\
Average & $F(7)=8.42, p=4.9 \times 10^{-10}$ \\
Fractional anisotropy & $F(7)=6.99, p=3.7 \times 10^{-8}$ \\
Gaussian & $F(7)=7.36, p=1.2 \times 10^{-8}$ \\
Streamline counts & $F(7)=0, p=1.00$ \\
Power-law & $F(7)=189.11, p=3.9 \times 10^{-179}$ \\
Modal & $F(7)=182.97, p=4.6 \times 10^{-175}$ \\
Fractional anisotropy & $F(7)=1426.98, p=0.00$ \\
Gaussian & $F(7)=0, p=1.00$ \\
Streamline counts & \\
Power-law & $F(7)=237.7, p=6 \times 10^{-209}$ \\
Boundary & $F(7)=253.1, p=1 \times 10^{-217}$ \\
Fractional anisotropy & $F(7)=48.9, p=5 \times 10^{-60}$ \\
Gaussian & $F(7)=149.57, p=2 \times 10^{-151}$ \\
\hline Poweamline counts &
\end{tabular}

Results of one-way ANOVAs assessing the effect of graph model on each controllability statistic, and each edge weighting scheme, as shown in the boxplots in Figs. 9, 10, 11, and 12

controllability values, low variance (BA model lowest), and skewed left-tailed distributions toward lower controllability values (Fig. 11b,d). The power-law-weighted graphs had lower variance and slightly higher mean than those of the graphs weighted by streamline counts. One-way ANOVAs indicated significant effects of graph type for three of the four edge weight distributions: FA, streamline counts, and Gaussian but not power-law (see Table 5).

Finally, when considering boundary controllability for graph models constructed with a Gaussian weighting scheme, we observed that RL, WS, MD2, MD4, RG, and BA had right-tailed distributions, with increasing variance and skewness from RL to WS, MD2, RG, and BA. MD4 was also right-skewed and had the lowest variance. WRG and MD8 were not skewed toward higher controllability values, had more symmetric distributions, and had lower variance than the other graph types. The boundary controllability values of the graph types with FA weighting and power-law weighting followed similar trends to those observed with the Gaussian weighting. The differences were that, for power-law weighting, the overall controllability values were an order of magnitude smaller than those of Gaussian weighting and the MD4 graph had higher variance. For FA weighting, RL, WS, and MD4 all had lower variance than for Gaussian weighting. The graphs weighted by streamline counts exhibited a 

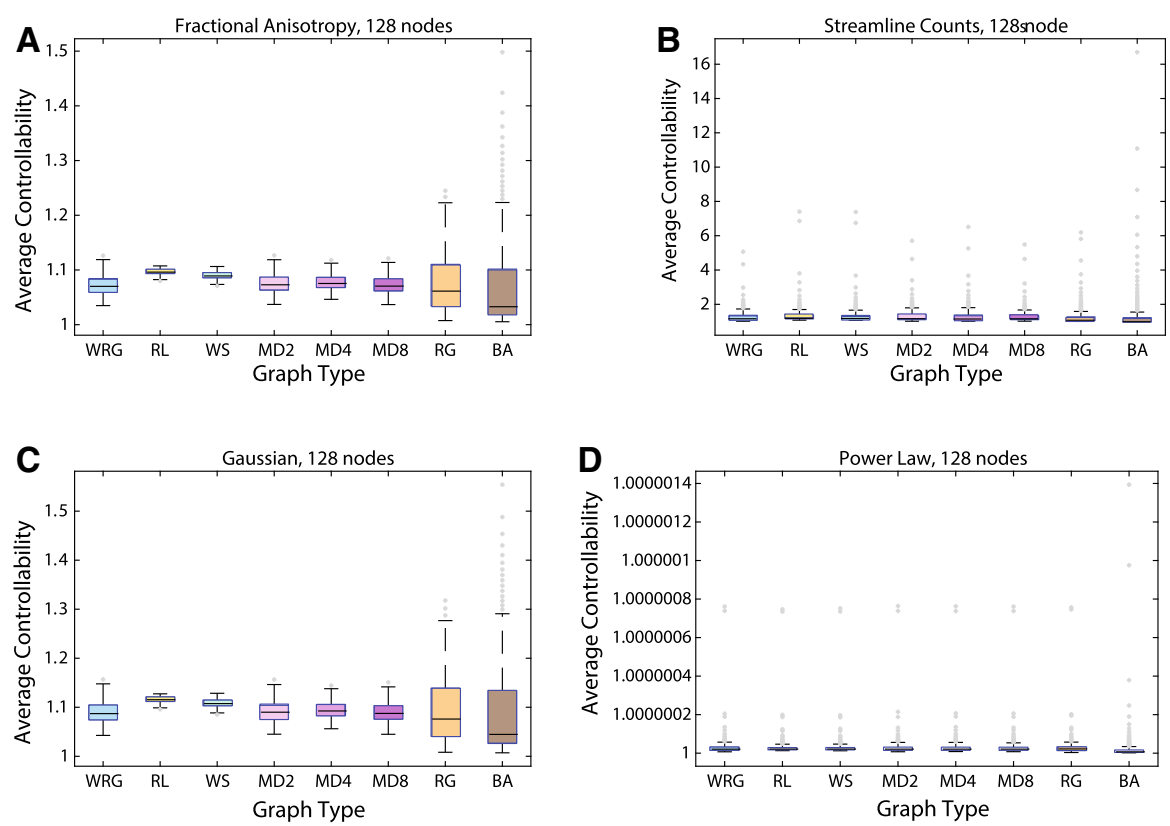

Fig. 10 Nodal variation in average controllability as a function of edge weighting and graph model. Average controllability values were averaged over instances in each graph model ensemble, and therefore, boxplots show variation over nodes in the graph. Results are shown for four edge weighting schemes: a FA, b streamline counts, c Gaussian, d power-law. The eight graph models include the weighted random graph (WRG), the ring lattice (RL), the Watts-Strogatz small-world (WS), the modular graphs (MD2, MD4, MD8), the random geometric (RG), and the Barábasi-Albert preferential attachment (BA) models

different trend, with low means (all between 0 and 1), low variance, and slight righttailed distributions, with the BA graph being the most skewed. One-way ANOVAs indicated significant effects of graph type for all of the four edge weight distributions (see Table 5).

To summarize, when considering trends within a single edge weighting scheme, it is important to note that because edge weight distributions were exactly the same across each of the graph types, this guarantees that differences in controllability are due to network topology rather than the effects of differing edge weights. Since each of the graph types exhibits distinct behavior of controllability values for all types of controllability except average and modal controllability in power-law-weighted graphs, this suggests that the topology of a network largely influences global, average, modal, and boundary controllability, but these influences can be obfuscated in graphs whose edge weights follow a power-law distribution.

\subsection{Relation Between Controllability Statistics Across Nodes}

In prior work, we have observed that average and modal controllability are inversely related to one another across regions in brain networks, both in noninvasive human neuroimaging data acquired in youth and adults (Gu et al. 2015; Tang et al. 2017) 

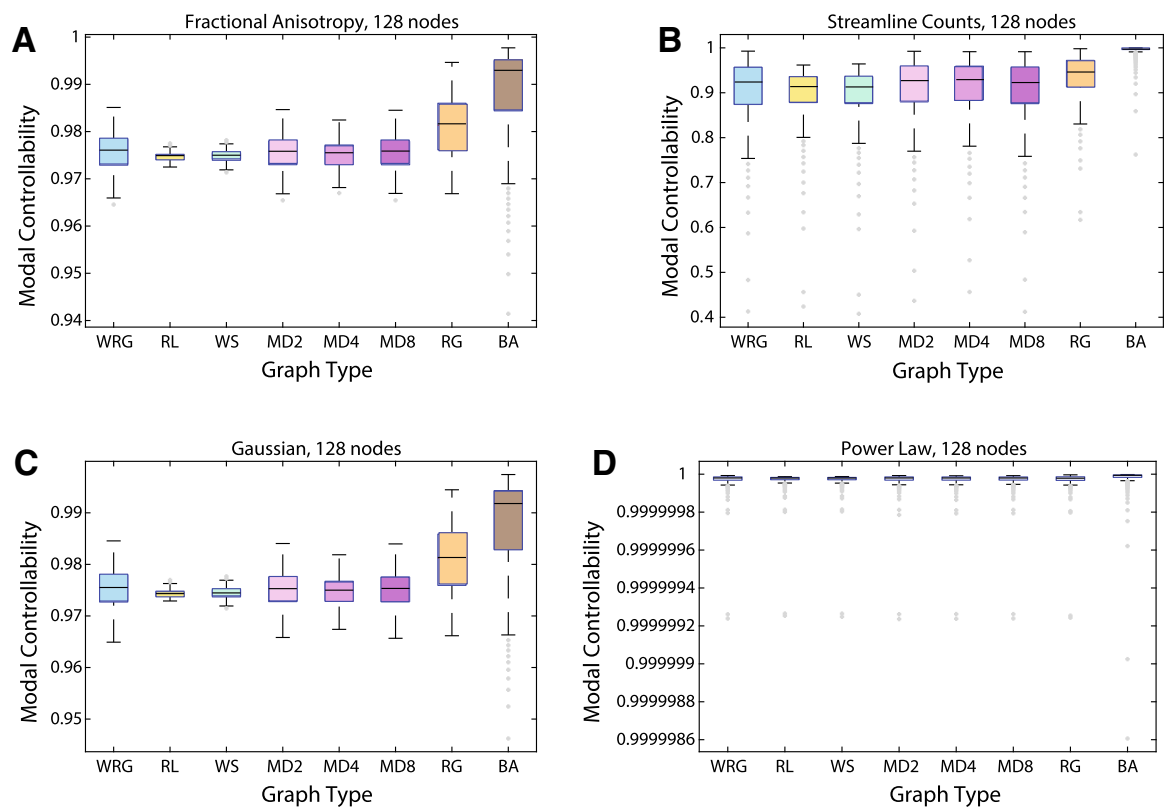

Fig. 11 Nodal variation in modal controllability as a function of edge weighting and graph model. Modal controllability values were averaged over instances in each graph model ensemble, and therefore, boxplots show variation over nodes in the graph. Results are shown for four edge weighting schemes: a FA, b streamline counts, c Gaussian, d power-law. The eight graph models include the weighted random graph (WRG), the ring lattice (RL), the Watts-Strogatz small-world (WS), the modular graphs (MD2, MD4, MD8), the random geometric (RG), and the Barábasi-Albert preferential attachment (BA) models

and in tract-tracing data acquired from macaque monkeys ( $\mathrm{Gu}$ et al. 2015). These data suggest that regions of the brain that are structurally predisposed to be effective in moving the brain into easy-to-reach states (via average control) are different from the regions of the brain that are structurally predisposed to be effective in moving the brain into difficult-to-reach states (via modal control). Here, we ask whether this inverse relationship between average and modal controllability across nodes holds in canonical graph models.

For graphs constructed with the Gaussian weighting scheme, we observed that average and modal controllability were negatively correlated with one another across all 8 graph models, with RL having the weakest and BA having the strongest correlation (Fig. 13). We observed similar trends in the graphs constructed with the FA weighting scheme, while in graphs constructed with the streamline count weighting scheme we observed the BA graph model to have the strongest correlation, and in the powerlaw-weighting scheme we observed that the relationship between average and modal controllability was nearly perfectly linear across all graph models (see Supplementary Materials). For Spearman $\rho$ correlation coefficients, see Table 6.

In prior work, we have observed that average and boundary controllability are not strongly related to one another across regions in adult human brain networks (Gu et al. 2015). These data suggest that regions of the brain that are structurally 

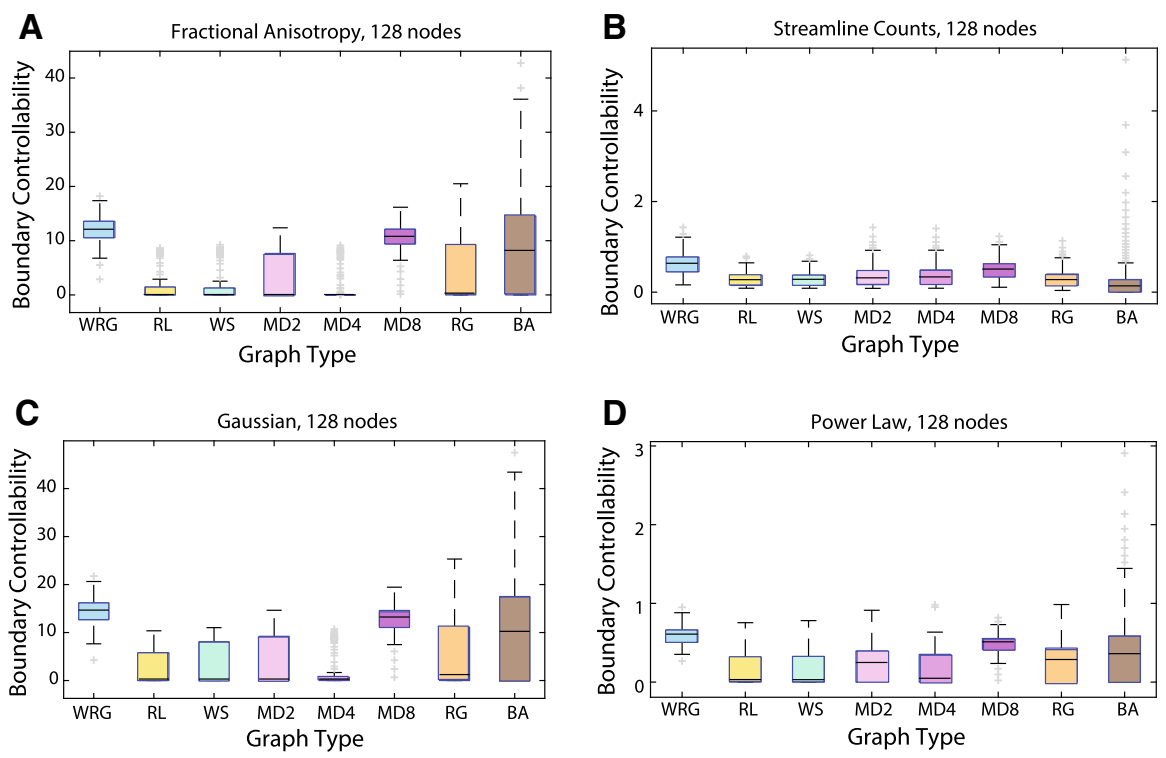

Fig. 12 Nodal variation in boundary controllability as a function of edge weighting and graph model. Boundary controllability values were averaged over instances in each graph model ensemble, and therefore, boxplots show variation over nodes in the graph. Results are shown for four edge weighting schemes: a FA, b streamline counts, $\mathbf{c}$ Gaussian, $\mathbf{d}$ power-law. The eight graph models include the weighted random graph (WRG), the ring lattice (RL), the Watts-Strogatz small-world (WS), the modular graphs (MD2, MD4, MD8), the random geometric (RG), and the Barábasi-Albert preferential attachment (BA) models. Note that these results are presented for $\rho=10^{-8}$; we observe consistent results across percentiles of $10^{-5}$, $10^{-8}$, and $10^{-11}$ of edge weight distribution (see Supplement)
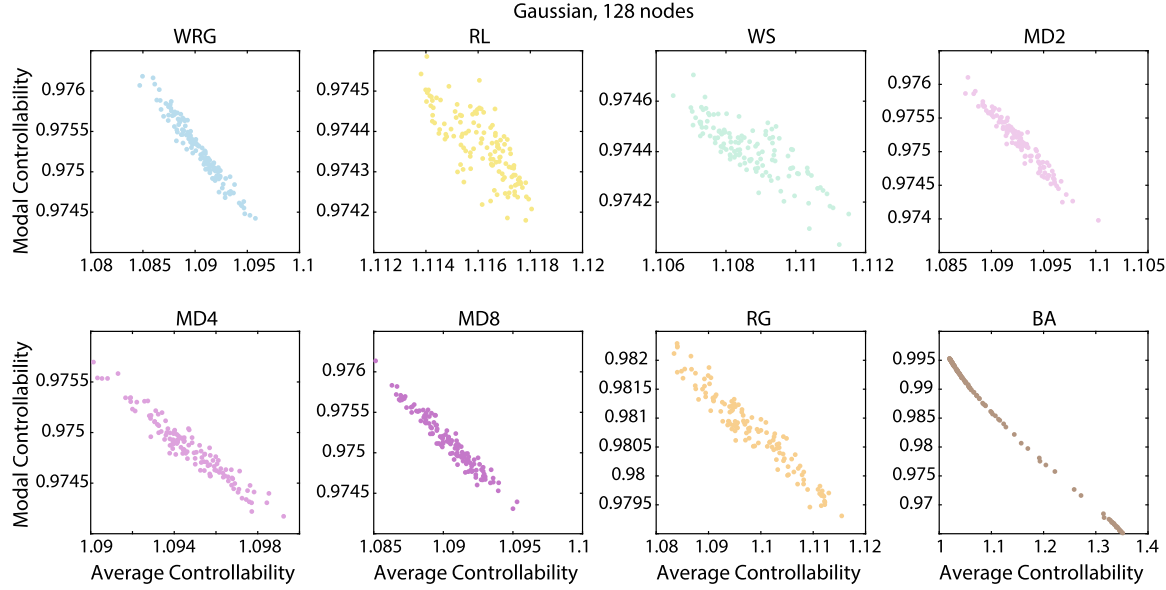

Fig. 13 Relation between average and modal controllability across nodes for the Gaussian edge weighting scheme. Average and modal controllability values were averaged over instances in each graph model ensemble, and therefore, scatterplots show values for each node in the graph. Results are shown for the Gaussian edge weighting scheme. The eight graph models include the weighted random graph (WRG), the ring lattice (RL), the Watts-Strogatz small-world (WS), the modular graphs (MD2, MD4, MD8), the random geometric $(\mathrm{RG})$, and the Barábasi-Albert preferential attachment (BA) models 


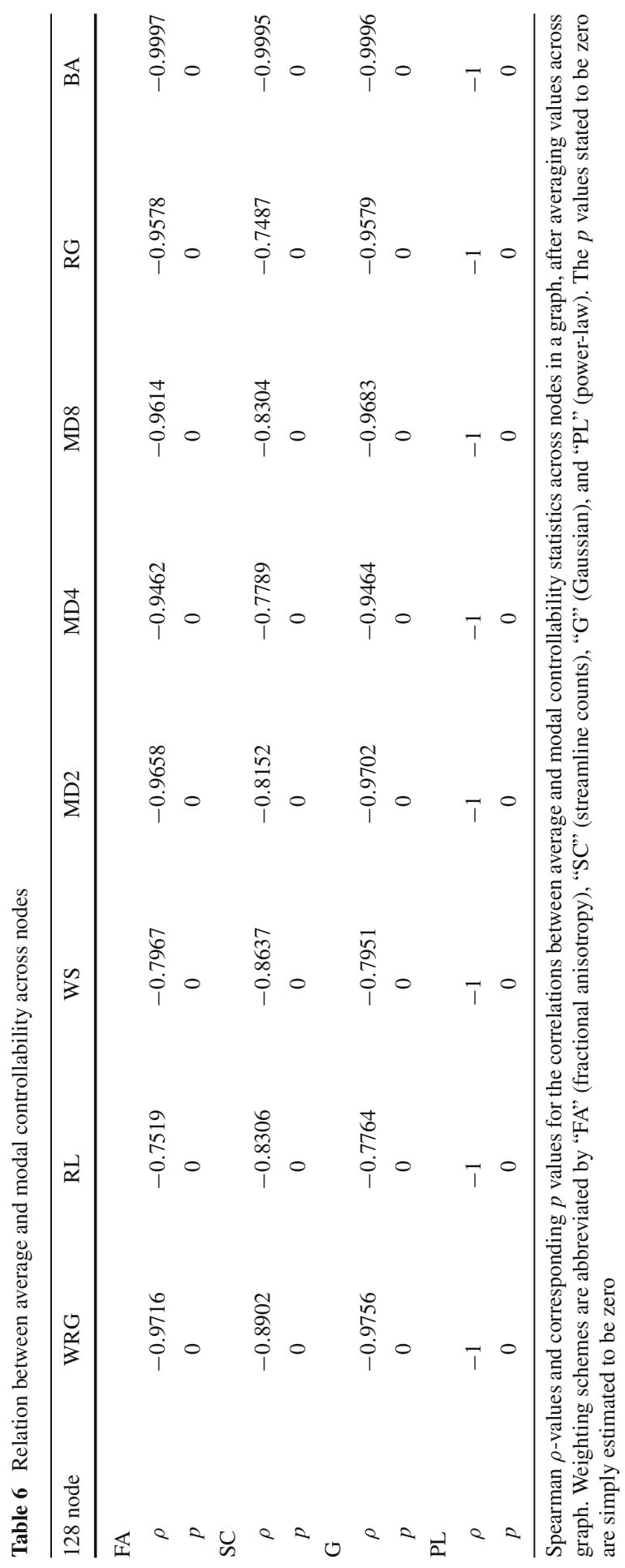



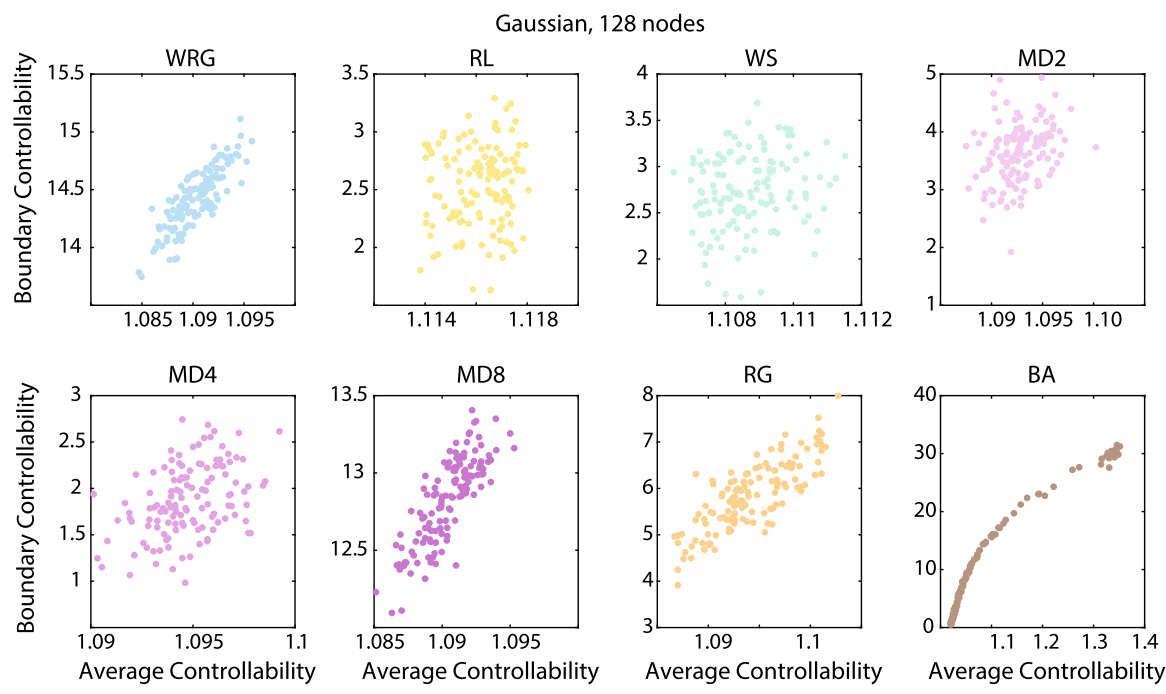

Fig. 14 Relation between average and boundary controllability across nodes for the Gaussian edge weighting scheme. Average and boundary controllability values were averaged over instances in each graph model ensemble, and therefore, scatterplots show values for each node in the graph. Results are shown for the Gaussian edge weighting scheme. The eight graph models include the weighted random graph (WRG), the ring lattice (RL), the Watts-Strogatz small-world (WS), the modular graphs (MD2, MD4, MD8), the random geometric (RG), and the Barábasi-Albert preferential attachment (BA) models

predisposed to be effective in moving the brain into easy-to-reach states (via average control) may sometimes (but not consistently) also be the regions of the brain that are structurally predisposed to be effective in gating across network communities (via boundary control). Here, we ask whether the lack of a relationship between average and boundary controllability across nodes holds in canonical graph models. In general for Gaussian weighting schemes, we observe that average controllability tends to be positively correlated with boundary controllability across nodes (Fig. 14); in contrast, boundary controllability tends to be negatively correlated with modal controllability across nodes (Fig. 15). These relationships appear to be least salient in small-world and modular graphs, which may explain why they were not previously observed in brain networks (Gu et al. 2015). Across other weighting schemes, we observe consistent trends, for average controllability to be positively related to boundary controllability, and for modal controllability to be negatively related to boundary controllability across nodes in a graph (Tables 7 and 8).

\subsection{Effect of Graph Size on Nodal Variation in Network Controllability Statistics Across Graph Models}

To assess the reliability and reproducibility of our results, we next examined the impact of graph size ( $n=128,256$, or 512$)$ on network controllability statistics, and their modulation by graph model for the Gaussian edge weight distribution (see Supplementary Materials). We observed that global, average, modal, and boundary controllability val- 
Gaussian, 128 nodes
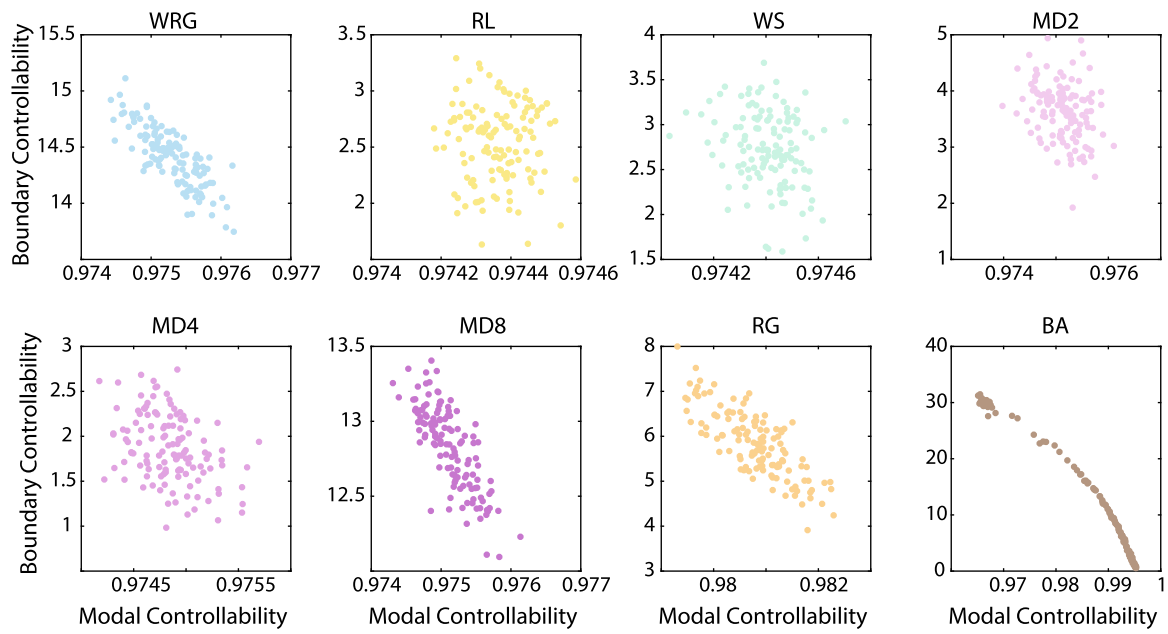

Fig. 15 Relation between modal and boundary controllability across nodes for the Gaussian edge weighting scheme. Modal and boundary controllability values were averaged over instances in each graph model ensemble, and therefore, scatterplots show values for each node in the graph. Results are shown for the Gaussian edge weighting scheme. The eight graph models include the weighted random graph (WRG), the ring lattice (RL), the Watts-Strogatz small-world (WS), the modular graphs (MD2, MD4, MD8), the random geometric (RG), and the Barábasi-Albert preferential attachment (BA) models

Table 7 Relation between average and boundary controllability across nodes

\begin{tabular}{cllllllll}
\hline 128 node & WRG & RL & WS & MD2 & MD4 & MD8 & RG & BA \\
\hline FA & & & & & & & & \\
$\rho$ & 0.7915 & 0.1495 & 0.1246 & 0.3393 & 0.4141 & 0.7464 & 0.7816 & 0.9939 \\
$p$ & 0 & 0.0921 & 0.1609 & 0.0001 & 0.0000 & 0 & 0 & 0 \\
SC & & & & & & & & \\
$\rho$ & 0.3324 & -0.0475 & -0.1106 & 0.3404 & 0.2763 & -0.0041 & 0.1169 & 0.9935 \\
$p$ & 0.0001 & 0.5944 & 0.2136 & 0.0001 & 0.0017 & 0.9634 & 0.1887 & 0 \\
G & & & & & & & & \\
$\rho$ & 0.8217 & 0.0665 & 0.1727 & 0.3213 & 0.4041 & 0.8181 & 0.7871 & 0.9960 \\
$p$ & 0 & 0.4551 & 0.0513 & 0.0002 & 0.0000 & 0 & 0 & 0 \\
PL & & & & & & & & \\
$\rho$ & 0.3400 & 0.5281 & 0.5678 & 0.4121 & 0.5852 & -0.1224 & 0.3145 & 0.9970 \\
$p$ & 0.0001 & 0.0000 & 0 & 0.0000 & 0 & 0.1683 & 0.0003 & 0 \\
\hline
\end{tabular}

Spearman $\rho$-values and corresponding $p$ values for the correlations between average and boundary controllability statistics across nodes in a graph, after averaging values across graph. Weighting schemes are abbreviated by "FA" (fractional anisotropy), "SC" (streamline counts), "G" (Gaussian), and "PL" (power-law). The $p$ values stated to be zero are simply estimated to be zero 


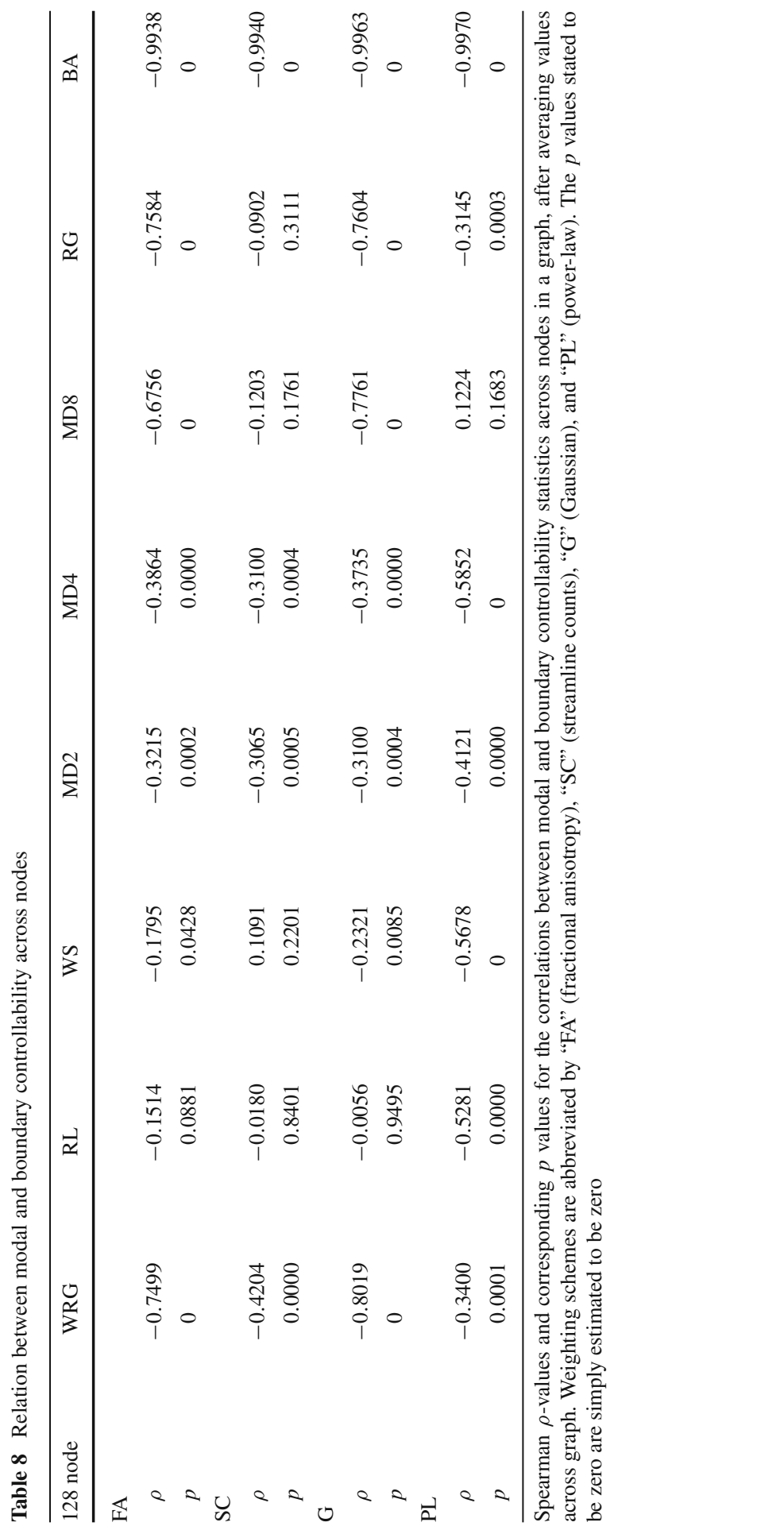


A

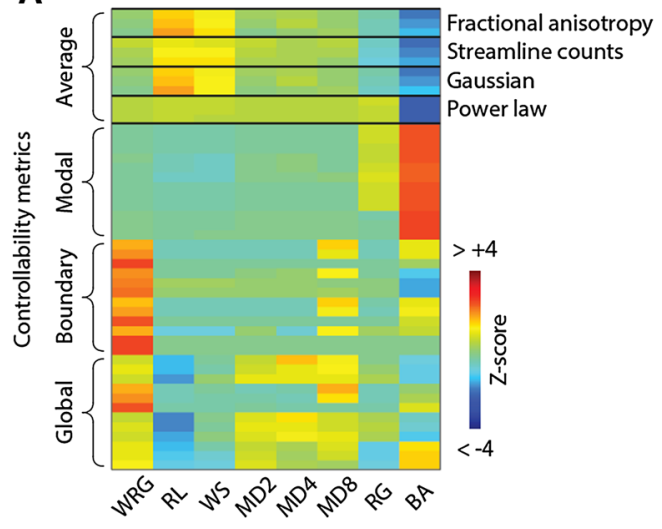

B

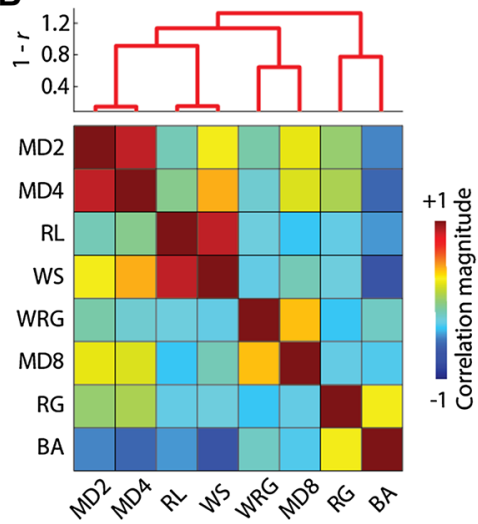

Fig. 16 Clustering controllability features across graph models. For each graph model, we extracted the median average, modal, boundary, and global controllability statistics for the four edge weight distributions and graphs of 128, 256, and 512 nodes. a Matrix of controllability statistics $z$-scored across graph models so that each row has a mean of zero and unit variance. b We computed the Pearson correlation coefficient, $r$, of controllability metrics for every pair of graphs and clustered that matrix, revealing graph types whose controllability statistics were correlated across the manipulations studied in this report

ues for graphs of 256 and 512 nodes largely maintained the same trends as for graphs with 128 nodes. However, mean global controllability values tended to decrease with increasing graph size, and the variance of average, modal, and boundary controllability values tended to decrease with increasing graph size. In addition, for the MD8 graph, the mean boundary controllability decreases to close to 0 in the graph of size 512 . Importantly, because size varied within the same type of controllability for the same set of graphs, this guarantees that differences in controllability are due to the effects of differing network size rather than network topology. The similarity of trends across graph sizes suggests that the controllability properties are maintained in networks of different sizes but may be accentuated through decreased spread or mean value with increasing size.

\subsection{Similarities in Patterns of Controllability Statistics Between Graph Models}

Finally, we asked whether certain graphs with similar topologies might show similar patterns of controllability statistics across edge weighting schemes and network sizes. To address this question, we treated average, modal, boundary, and global controllability as features of interest, and extracted their median values for each of the eight graph models. Rather than express these statistics as raw scores which can sometimes differ by many orders of magnitude, we standardized them across graph models and expressed them as $z$-scores. We repeated this process for all combinations of edge weight distributions and graph sizes, resulting in 48 features for each graph model (Fig. 16a).

To assess the similarity of controllability statistics across graph models, we computed the model-by-model correlation matrix of $z$-scored features (Fig. 16b). This 
matrix was marked by heterogeneity. Intuitively, we observed that networks with similar topological features exhibited similar behavior in terms of their controllability statistics. For example, the two- and four-module graphs, which both featured large, segregated modules, displayed patterns of statistics that were highly correlated with one another $\left(r=0.86, p<10^{-14}\right)$. Likewise, the ring lattice and the Watts-Strogatz graphs, which featured highly regular organization, displayed patterns of statistics that were also highly correlated $(r=0.85, p<10-14)$. Not surprisingly, the BarábasiAlbert model, which was the only model we included that had a heavy-tailed degree (and strength) distribution, was dissimilar to the other models, on average. These findings suggest that network topological properties induce similarities in the behavior of controllability statistics across graph models.

\section{Discussion}

The representation of complex systems as graphs or networks has proven useful in a wide variety of domains for the study of physical (Papadopoulos et al. 2016), technological (Tan et al. 2014), biological (Steinway et al. 2015; Baldassano and Bassett 2016), ecological (Proulx et al. 2005), and social (Ilany and Akcay 2016; Evans et al. 2016) processes. While initial work in each of these domains focused on developing descriptive statistics to characterize the nature of the system's graph representation, more recent efforts have turned to developing predictions of system function and fundamental theories of system dynamics (Bassett and Sporns 2017). One particularly powerful approach to both prediction and theory for these systems comes from the emerging field of network control, which provides analytical results for the response of a network system to internal or external perturbation (Bassett et al. 2017). The application of these tools to neural systems has recently provided important insights into possible mechanisms of cognitive control ( $\mathrm{Gu}$ et al. 2015), energetic explanations for baseline activation of the default mode system (Betzel et al. 2016), and the emergence of diverse dynamics over the course of normative neurodevelopment (Tang et al. 2017).

Despite these initial successes, a basic understanding of the performance of these tools on graphs with different topologies or geometries is lacking (Bianchin et al. 2015). Here we address this challenge by studying commonly applied network controllability statistics assuming linear system dynamics to several canonical graph models built with distinct edge weight distributions. We find that both graph topology and edge weight distribution can impact network controllability statistics estimated at single regions or across the whole network. These data underscore the importance of assessing network controllability statistics in one's own data (as well as statistic-statistic relations) rather than relying on assumptions built from other data. More importantly, the results point to the necessity of developing analytical descriptions of the relations between topology, geometry, and control (Kim et al. 2018).

\subsection{Understanding Topological Drivers of Control}

Intuitively, one might imagine that the topology of a graph should have a non-trivial impact on the types of control strategies that the system can perform or respond to. 
A star graph, containing many nodes only (and directly) connected to a single central node, may have quite different responses to energy injected into the central node than to energy injected into the peripheral nodes (Pasqualetti and Zampieri 2014). Moreover, both of these responses may be quite distinct from the response of a lattice graph to local perturbations at any of the nodes (Bianchin et al. 2015). The intuition that topology matters for control is one that has now been supported by decades of prior literature (Reinschke 1988) and is quantitatively demonstrated in our results, which show that canonical graph models tend to display significantly different values for global, average, modal, and boundary controllability, both in variations across a graph ensemble and in variations across network nodes. This intuition has further motivated both the development of controllability statistics (Pasqualetti et al. 2014) and of intuitions for how controllability may relate to the system's dynamics (synchronizability) and geometry (symmetry; Whalen et al. (2015)). Recent work has pinpointed specific features of edge weight vectors associated with each node that explain the energy expected for control (Kim et al. 2018), and this work also suggested that these features vary in a meaningful way over species whose origins span evolutionary time scales.

Collectively, these studies motivate the question of whether neural systems have a characteristic graph topology that supports their specific functions as information processing systems in organisms. Canonical graph models with simple rules specifying the existence or growth of connections have proven useful in initial forays into this question (Klimm et al. 2014; Samu et al. 2014). While evidence suggests that region location (Kaiser and Hilgetag 2006), spatial embedding (Samu et al. 2014), and mechanisms for growth (Klimm et al. 2014) are important drivers of graph topology in neural systems, most graph models offer reasonable explanations for only one or a very few characteristic features of neural networks. Canonical graph models are therefore commonly used as benchmarks against which to compare real-world topologies, rather than as exact replicas of the biological system under study. As benchmarks, it is important to understand the expected controllability profiles of these graph modelsas we do here-so as to inform interpretations regarding the biological specificity of control profiles observed empirically. Indeed, our observations will be useful in determining the degree to which simple connection rules (including random, smallworld, and preferential attachment) can account for controllability statistics in neural systems.

\subsection{Non-trivial Impact of Edge Weight Distributions on Controllability of Weighted Networks}

An important contribution of our work stems from the fact that we do not study binary graph models but instead examine graphs that have been weighted by drawing from statistical and empirical functional forms for edge weight distributions. Our choice to focus on weighted graph models was motivated by recent work, demonstrating that assessing binary versions of weighted graphs can provide inaccurate intuitions regarding a network's architecture, and by extension its function (Bassett and Bullmore 2016). For example, quite dense graphs can appear to lack small-world architecture 
if studied as a binary matrix but display strong small-world architecture when edge weights are taken into account (Muldoon et al. 2016). This discrepancy can be understood when considering the fact that weighting schemes tell us about the geometry (weight distribution and weight location) of a network (Bassett et al. 2012). Importantly, edge weights can have a direct impact on the potential to control and on the energy required for control of neural systems (Kim et al. 2018). Here, we observe that edge weight distributions can either enhance or obfuscate differences in controllability profiles across graph models, either in variations across a graph ensemble and in variations across network nodes. These findings suggest that it is wise to be cautious about inferring the controllability profile of a graph model independent of knowledge regarding its edge weight distribution. They also suggest interesting future directions for network design, particularly in cases where the graph architecture is fixed by external constraints but where the edge weights can be varied with the goal of enhancing or decrementing control.

\subsection{Relations Between Controllability Statistics Prescribed By Graph Topology}

Initial efforts focused on a narrow class of graphs showed that average and modal controllability were positively correlated with one another across graphs instances (Tang et al. 2017), and negatively correlated with one another across nodes ( $\mathrm{Gu}$ et al. 2015), while average and boundary controllability were not significantly correlated with one another across nodes. Here we show that only one of these observations holds true across both graph models and edge weight distributions: that average and modal controllability are correlated with one another across nodes. In contrast to prior work in brain graphs, we show that average and modal controllability can be positively, negatively, or nonsignificantly correlated with one another across graph instances in an ensemble and that boundary controllability tends to be positively correlated with average controllability (and negatively correlated with modal controllability) across nodes in a graph. These data suggest that the relationship between controllability statistics depends strongly on the graph model's topological architecture and on the observed edge weight distribution. These findings are interesting because they suggest the possibility of designing networks with different strengths for one type of control versus another or for specific relationships between control profiles. Such a possibility is further bolstered by the fact that we observe in a hierarchical clustering procedure that certain graph models share greater similarity in their entire profile of controllability statistics (across the dimensions of size, edge weight distribution, etc. studied here) to some graph models than to other graph models.

\subsection{Methodological Considerations}

There are several methodological considerations that are pertinent to this work. First, we note that the network controllability statistics that we study are based fundamentally on a linear model of dynamics (Pasqualetti et al. 2014). Such a model is clearly appropriate for linear systems, but its application to systems characterized by nonlinear dynamics must be considered carefully. Practically speaking, linear models can 
provide excellent predictive power for a system in the neighborhood of the operating point (Leith and Leithead 2000). In the context of neural systems, linear models of dynamics have proven useful both at the ensemble level and at the large-scale regional level in predicting intrinsic dynamics (Galán 2008; Honey et al. 2009). Moreover, linear predictions of response to control input have been validated in nonlinear models of cortical columns (Muldoon et al. 2016). These studies support the investigation of linear control in neural systems, but do not preclude future studies of explicitly nonlinear control (Cornelius et al. 2013; Motter 2015), which could also prove useful in understanding neural systems (Tang and Bassett 2017).

A second important consideration relates to the control strategies that we study: global, average, modal, and boundary controllability. While these notions have proven useful both in man-made (Pasqualetti et al. 2014) and natural (Gu et al. 2015) systems, they are by no means an exhaustive list of the sorts of measures that may be interesting to study in canonical graph models. We chose them for their prior application to brain networks, which have motivated the choices of edge weight distributions in this study. However, other interesting approaches include the identification of driver nodes (Liu et al. 2011), the estimate of control energy used for specific types of state transitions (Betzel et al. 2016; Gu et al. 2017), the susceptibility of the system to compensatory perturbations (Cornelius et al. 2013), as well as the measurement of the controllability radius (Menara et al. 2018), structural permeability (Lo Iudice et al. 2015), edge controllability (Pang et al. 2017), and others. Moreover, in addition to these previously defined measures, it is intuitively plausible that other as-yet-undefined control strategies may also prove relevant. Indeed, the definition of control metrics for complex networks has received increasing attention in recent years (Pasqualetti et al. 2014), and future work is likely to develop a wider battery of statistics.

A third important consideration relates to limitations of the data that we used to construct our empirical edge weight distributions. Diffusion imaging is a powerful noninvasive neuroimaging technique (Wiegell et al. 2000), which has only recently become commonplace in the construction of human (and non-human) connectomes (Hagmann et al. 2010; Johansen-Berg and Rushworth 2009). The technique is relatively new, and tractography algorithms applied to the data continue to be refined (Jbabdi and Johansen-Berg 2011; Pestilli et al. 2014). It will be important in future work to evaluate other empirically estimated edge weight distributions as they become available.

A fourth point is that we chose a specific normalization factor for our matrices to ensure stability. However, we note that different choices of normalization may accentuate versus de-emphasize different scales of dynamics, and it will be interesting in the future to study how the choice of normalization impacts observed patterns of controllability.

A fifth point is that we study controllability from a single node only. However, methods do exist for studying multi-point control (Betzel et al. 2016; Gu et al. 2017), and an important future direction for research is to understand how graph models differ in their capacities for multi-point control. Indeed, the global and average controllability metrics (though not the modal and boundary controllability metrics) that we study here are defined for arbitrary $\mathbf{A}$ and $\mathbf{B}$ and therefore are amenable to studies of both single-point and multi-point control. Here, our focus on single-node control is justified 
because we are interested in node variability, and not on specific controllability values per say. However, it is fair to say that we do not yet know whether the intuitions that we gain from single-point control are relevant for multi-point control because, even though the dynamics are linear, the effects of multi-point control are not generally a linear function of the corresponding single-node controllability measures.

A sixth point is that the computation of the controllability Gramian is intrinsically difficult because its smallest eigenvalue is in fact very small. Here we report the estimated numerical values and statistics and only interpret differences in ensembles of graphs, and not differences between pairs of graphs. We also acknowledge that variations of the controllability values are often small and that the importance of this variation likely depends on the specific application domain. Indeed, ongoing work outside of the scope of this study is addressing questions of appropriate normalization depending on the network size, the distribution of edge weights, and the number of control points being examined. Nevertheless, the numerical results highlight important trends and dependencies, which are independent of the actual numerical value.

A final important consideration relates to the set of graph models that we study. While we cover many of the canonical models that are frequently studied in network science, and especially those more frequently studied in the context of human and non-human brain networks (Klimm et al. 2014; Sizemore et al. 2017), the set is in no way exhaustive. It would be interesting in future work to further develop biologically motivated growth models that may more accurately take into account the neurophysiological processes of cell migration, synaptic plasticity and pruning, and other mutually trophic influences on neural development.

\section{Conclusion}

In conclusion, we here examine a set of statistics that characterize diverse control strategies of networked systems whose dynamics (at least in a particular regime) can be approximated by a linear, noise-free, discrete-time, and time-invariant model. We apply these statistics to graph models whose edge weights are drawn from both empirical and statistically defined functional forms. We show that controllability metrics, and their relations to one another, differ across graph models, and that those relations can be either elucidated or clouded by the distribution of edge weights in the graph. We observe that modular graph models show the most positively correlated patterns of controllability values across network size, controllability statistic, and edge weight distribution, while the Watts-Strogatz small-world model and Barábasi-Albert preferential attachment model show the most negatively correlated patterns. Our study offers intuitions for how controllability statistics behave in common graph models used as benchmarks for studies of brain networks in both human and non-human species. More generally, it suggests interesting future directions in designing networks to display a pattern of controllability statistics (and relations between them), particularly when the graph architecture is fixed by external constraints but the edge weights can be varied to enhance or decrement control. 
Acknowledgements DSB would like to acknowledge support from the John D. and Catherine T. MacArthur Foundation, the Alfred P. Sloan Foundation, the Army Research Laboratory and the Army Research Office through Contract Numbers W911NF-10-2-0022 and W911NF-14-1-0679, the National Institute of Health (2-R01-DC-009209-11, 1R01HD086888-01, R01-MH107235, R01-MH107703, R01MH109520, 1R01NS099348 and R21-M MH-106799), the Office of Naval Research, and the National Science Foundation (BCS-1441502, CAREER PHY-1554488, BCS-1631550, and CNS-1626008). FP would like to acknowledge support from NSF-BCS-1631112 and NSF-BCS-1430279. The content is solely the responsibility of the authors and does not necessarily represent the official views of any of the funding agencies.

Open Access This article is distributed under the terms of the Creative Commons Attribution 4.0 International License (http://creativecommons.org/licenses/by/4.0/), which permits unrestricted use, distribution, and reproduction in any medium, provided you give appropriate credit to the original author(s) and the source, provide a link to the Creative Commons license, and indicate if changes were made.

\section{References}

Baldassano, S.N., Bassett, D.S.: Topological distortion and reorganized modular structure of gut microbial cooccurrence networks in inflammatory bowel disease. Sci. Rep. 6, 26087 (2016)

Bassett, D.S., Bullmore, E.T.: Small-world brain networks revisited. Neuroscientist Sep 21, $1073858416667720(2016)$

Bassett, D.S., Khambhati, A.N., Grafton, S.T.:Emerging frontiers of neuroengineering: a network science of brain connectivity. Annu. Rev. Biomed. Eng. 27 (2017)

Bassett, D.S., Sporns, O.: Network neuroscience. Nat. Neurosci. 20, 353-364 (2017)

Bassett, D.S., Owens, E.T., Daniels, K.E., Porter, M.A.: Influence of network topology on sound propagation in granular materials. Phys. Rev. E Stat. Nonlinear Soft Matter Phys. 86, 041306 (2012)

Bettencourt, L.M., Stephens, G.J., Ham, M.I., Gross, G.W.: Functional structure of cortical neuronal networks grown in vitro. Phys. Rev. E Stat. Nonlinear Soft Matter Phys. 75, 021915 (2007)

Betzel, R.F., Gu, S., Medaglia, J.D., Pasqualetti, F., Bassett, D.S.: Optimally controlling the human connectome: the role of network topology. Sci. Rep. 6, 30770 (2016)

Betzel, R.F., Medaglia, J.D., Papadopoulos, L., Baum, G.L., Gur, R.C., Gur, R.E., Roalf, D., Satterthwaite, T.D., Bassett, D.S.: The modular organization of human anatomical brain networks: accounting for the cost of wiring. Netw. Neurosci. 1, 42-68 (2017)

Bianchin, G., Pasqualetti, F., Zampieri S.: The role of diameter in the controllability of complex networks. In: IEEE Conference on Decision and Control, pp. 980-985 (2015)

Boccaletti, S., Latora, V., Moreno, Y., Chavez, M., Hwang, D.-U.: Complex networks: structure and dynamics. Phys. Rep. 424, 175-308 (2006)

Carrington, P.J., Scott, J., Wasserman, S.: Models and Methods in Social Network Analysis, vol. 28. Cambridge University Press, Cambridge (2005)

Colizza, V., Barrat, A., Barthélemy, M., Vespignani, A.: The role of the airline transportation network in the prediction and predictability of global epidemics. Proc. Natl. Acad. Sci. U.S.A. 103, 2015-2020 (2006)

Cornelius, S.P., Kath, W.L., Motter, A.E.: Realistic control of network dynamics. Nat. commun. 4, 1942 (2013)

Evans, C.R., Onnela, J.P., Williams, D.R., Subramanian, S.V.: Multiple contexts and adolescent body mass index: schools, neighborhoods, and social networks. Soc. Sci. Med. 162, 21-31 (2016)

Galán, R.F.: On how network architecture determines the dominant patterns of spontaneous neural activity. PloS one 3, e2148 (2008)

Goh, K.-I., Cusick, M.E., Valle, D., Childs, B., Vidal, M., Barabási, A.-L.: The human disease network. In: Proceedings of the National Academy of Sciences, Vol. 104, pp. 8685-8690 (2007)

Goñi, J., van den Heuvel, M.P., Avena-Koenigsberger, A., de Mendizabal, N.V., Betzel, R.F., Griffa, A., Hagmann, P., Corominas-Murtra, B., Thiran, J.-P., Sporns, O.: Resting-brain functional connectivity predicted by analytic measures of network communication. In: Proceedings of the National Academy of Sciences, vol. 111, pp. 833-838 (2014)

Gu, S., Pasqualetti, F., Cieslak, M., Telesford, Q.K., Alfred, B.Y., Kahn, A.E., Medaglia, J.D., Vettel, J.M., Miller, M.B., Grafton, S.T., et al.: Controllability of structural brain networks. Nat. Commun. 6, 8414 (2015) 
Gu, S., Betzel, R.F., Mattar, M.G., Cieslak, M., Delio, P.R., Grafton, S.T., Pasqualetti, F., Bassett, D.S.: Optimal trajectories of brain state transitions. Neuroimage 148, 305-317 (2017)

Hagmann, P., Cammoun, L., Gigandet, X., Gerhard, S., Grant, P.E., Wedeen, V., Meuli, R., Thiran, J.P., Honey, C.J., Sporns, O.: MR connectomics: principles and challenges. J. Neurosci. Methods 194, 34-45 (2010)

Heaton, L., Obara, B., Grau, V., Jones, N., Nakagaki, T., Boddy, L., Fricker, M.D.: Analysis of fungal networks. Fungal Biol. Rev. 26, 12-29 (2012)

Henderson, J.A., Robinson, P.A.: Geometric effects on complex network structure in the cortex. Phys. Rev. Lett. 107, 018102 (2011)

Honey, C.J., Kötter, R., Breakspear, M., Sporns, O.: Network structure of cerebral cortex shapes functional connectivity on multiple time scales. Proc. Natl. Acad. Sci. 104, 10240-10245 (2007)

Honey, C.J., Sporns, O., Cammoun, L., Gigandet, X., Thiran, J.P., Meuli, R., Hagmann, P.: Predicting human resting-state functional connectivity from structural connectivity. Proc. Natl. Acad. Sci. U.S.A. 106, 2035-2040 (2009)

Ilany, A., Akcay, E.: Social inheritance can explain the structure of animal social networks. Nat. Commun. 7, 12084 (2016)

Jbabdi, S., Johansen-Berg, H.: Tractography: where do we go from here? Brain Connect. 1, 169-183 (2011)

Johansen-Berg, H., Rushworth, M.F.: Using diffusion imaging to study human connectional anatomy. Annu. Rev. Neurosci. 32, 75-94 (2009)

Kailath, T.: Linear Systems, vol. 1. Prentice-Hall, Upper Saddle River (1980)

Kaiser, M., Hilgetag, C.C.: Nonoptimal component placement, but short processing paths, due to longdistance projections in neural systems. PLoS Comput. Biol. 2, e95 (2006)

Kim, J.Z., Soffer, J.M., Kahn, A.E., Vettel, J.M., Pasqualetti, F., Bassett, D.S.: Role of graph architecture in controlling dynamical networks with applications to neural systems. Nat. Phys. 14, 91-98 (2018)

Klimm, F., Bassett, D.S., Carlson, J.M., Mucha, P.J.: Resolving structural variability in network models and the brain. PLoS Comput. Biol. 10, e1003491 (2014)

Leith, D.J., Leithead, W.E.: Survey of gain-scheduling analysis and design. Int. J. Control 73, 1001-1025 (2000)

Liu, Yang-Yu., Barabási, A.-L.: Control principles of complex systems. Rev. Mod. Phys. 88, 035006 (2016)

Liu, Y.-Y., Slotine, J.-J., Barabási, A.-L.: Controllability of complex networks. Nature 473, 167-173 (2011)

Lo Iudice, F., Garofalo, F., Sorrentino, F.: Structural permeability of complex networks to control signals. Nat. Commun. 6, 8349 (2015)

Menara, T., Katewa, V., Bassett, D.S., Pasqualetti, F.: The structured controllability radius of symmetric (brain) networks. In: American Control Conference Submitted (2018)

Motter, A.E.: Networkcontrology. Chaos 25, 097621 (2015)

Muldoon, S.F., Pasqualetti, F., Gu, S., Cieslak, M., Grafton, S.T., Vettel, J.M., Bassett, D.S.: Stimulationbased control of dynamic brain networks. PLoS Comput. Biol. 12, e1005076 (2016)

Muldoon, S.F., Bridgeford, E.W., Bassett, D.S.: Small-world propensity and weighted brain networks. Sci. Rep. 6, 22057 (2016)

Newman, M.E.J.: Spread of epidemic disease on networks. Phys. Rev. E 66, 016128 (2002)

Newman, M.E.J., Girvan, M.: Finding and evaluating community structure in networks. Phys. Rev. E 69, 026113 (2004)

Newman, M., Barabasi, A.-L., Watts, D.J.: The Structure and Dynamics of Networks. Princeton University Press, Princeton (2011)

Pang, S.P., Wang, W.X., Hao, F., Lai, Y.C.: Universal framework for edge controllability of complex networks. Sci. Rep. 7, 4224 (2017)

Papadopoulos, L., Blinder, P., Ronellenfitsch, H., Klimm, F., Katifori, E., Kleinfeld, D., Bassett, D.S.: Embedding of biological distribution networks with differing environmental constraints. arXiv preprint arXiv:1612.08058 (2016)

Papadopoulos, L., Puckett, J.G., Daniels, K.E., Bassett, D.S.: Evolution of network architecture in a granular material under compression. Phys. Rev. E 94, 032908 (2016)

Pasqualetti, F., Zampieri, S.: On the controllability of isotropic and anisotropic networks. In: IEEE Conference on Decision and Control, pp. 607-612 (2014)

Pasqualetti, F., Zampieri, S., Bullo, F.: Controllability metrics, limitations and algorithms for complex networks. IEEE Trans. Control Netw. Syst. 1, 40-52 (2014)

Pestilli, F., Yeatman, J.D., Rokem, A., Kay, K.N., Wandell, B.A.: Evaluation and statistical inference for human connectomes. Nat. Methods 11, 1058-1063 (2014) 
Porter, M.A., Gleeson, J.P.: Dynamical systems on networks: a tutorial. arXiv preprint arXiv:1403.7663 (2014)

Proulx, S.R., Promislow, D.E., Phillips, P.C.: Network thinking in ecology and evolution. Trends Ecol. Evol. 20, 345-353 (2005)

Ray, B., Ghedin, E., Chunara, R.: Network inference from multimodal data: a review of approaches from infectious disease transmission. J. Biomed. Inform. 64, 44-54 (2016)

Reinschke, K.J.: Multivariable Control: A Graph-Theoretic Approach. Springer, Berlin (1988)

Roberts, J.A., Perry, A., Lord, A.R., Roberts, G., Mitchell, P.B., Smith, R.E., Calamante, F., Breakspear, M.: The contribution of geometry to the human connectome. Neuroimage 124, 379-393 (2016)

Ruths, J., Ruths, D.: Control profiles of complex networks. Science 343, 1373-1376 (2014)

Samu, D., Seth, A.K., Nowotny, T.: Influence of wiring cost on the large-scale architecture of human cortical connectivity. PLoS Comput. Biol. 10, e1003557 (2014)

Schweitzer, F., Fagiolo, G., Sornette, D., Vega-Redondo, F., White, D.R.: Economic networks: What do we know and what do we need to know? Adv. Complex Syst. 12, 407-422 (2009)

Sizemore, A.E., Giusti, C., Bassett, D.S.: Classification of weighted networks through mesoscale homological features. J. Complex Netw. 5, 245-273 (2017)

Sporns, O., Betzel, R.F.: Modular brain networks. Annu. Rev. Psychol. 67, 613-640 (2016)

Steinway, S.N., Biggs, M.B., Jr Loughran, T.P., Papin, J.A., Albert, R.: Inference of network dynamics and metabolic interactions in the gut microbiome. PLoS Comput. Biol. 11, e1004338 (2015)

Sun, J., Motter, E.: Controllability transition and nonlocality in network control'. Phys. Rev. Lett. 110, 208701 (2013)

Tan, F., Wu, J., Xia, Y., Tse, C.K.: Traffic congestion in interconnected complex networks. Phys. Rev. E Stat. Nonlinear Soft Matter Phys. 89, 062813 (2014)

Tang, E., Bassett, D.S.: Control of dynamics in brain networks. arXiv 1701, 01531 (2017)

Tang, E., Giusti, C., Baum, G., Gu, S., Kahn, A.E., David, R., Moore, T.M., Ruparel, K., Gur, R.C., Gur, R.E., et al.: Developmental increases in white matter network controllability support a growing diversity of brain dynamics. Nat. Commun. 8(1), 1252 (2017)

Teller, S., Granell, C., De Domenico, M., Soriano, J., Gomez, S., Arenas, A.: Emergence of assortative mixing between clusters of cultured neurons. PLoS Comput. Biol. 10, e1003796 (2014)

Whalen, A.J., Brennan, S.N., Sauer, T.D., Schiff, S.J.: Observability and controllability of nonlinear networks: the role of symmetry. Phys. Rev. X 5, 011005 (2015)

Wiegell, M.R., Larsson, H.B., Wedeen, V.J.: Fiber crossing in human brain depicted with diffusion tensor MR imaging. Radiology 217, 897-903 (2000)

Wiles, L., Gu, S., Pasqualetti, F., Parvesse, B., Gabrieli, D., Bassett, D.S., Meaney, D.F.: Autaptic connections shift network excitability and bursting. Sci. Rep. 7, 44006 (2017)

Yan, G., Ren, J., Lai, Y.-C., Lai, C.-H., Li, B.: Controlling complex networks: how much energy is needed? Phys. Rev. Lett. 108, 218703 (2012) 